\title{
Synthesis of functionalized pyridinium salts bearing a free amino group
}

\author{
Johanna Auth, ${ }^{a}$ Pablo Mauleón, ${ }^{b}$ and Andreas Pfaltz*a \\ ${ }^{a}$ Department of Chemistry, University of Basel, St. Johanns-Ring 19, \\ CH-4056 Basel, Switzerland \\ ${ }^{b}$ Departamento de Química Orgánica, Universidad Autónoma de Madrid, \\ Cantoblanco 28049, Spain \\ E-mail: andreas.pfaltz@unibas.ch
}

Dedicated to Professor Pierre Vogel on the occasion of his $\mathbf{7 0}^{\text {th }}$ birthday

DOI: $\underline{\text { http://dx.doi.org/10.3998/ark.5550190.p008.398 }}$

\begin{abstract}
Tetrasubstituted $\mathrm{N}$-methylpyridinium salts bearing a free tertiary amino group have been synthesized by a straightforward procedure starting from inexpensive starting materials. The key feature of the synthesis is the use of a proton as a simple effective protecting group to achieve selective $N$-methylation of the pyridine ring without attacking the amino group.
\end{abstract}

Keywords: Pyridinium salts, heterocycles, alkylations, pyridine $N$-methylation

\section{Introduction}

In metabolism, $\mathrm{NADH}$ is involved in redox reactions that transfer a hydride from one molecule to another. This coenzyme can be found in both its oxidized form $\left(\mathrm{NAD}^{+}\right)$and its reduced form $(\mathrm{NADH})$, which can be used as a reducing agent. The very selective nature of NADH-based reductions has inspired the development of a number of synthetic functional analogues. ${ }^{1}$ Introduction of functional groups that can participate in hydride transfer reactions offers the potential to induce new reactivity and selectivity patterns. However, the development of methods for the preparation of such analogues is hampered by the relatively high electrophilicity of pyridines and pyridinium salts, ${ }^{2-4}$ which significantly complicates attempts to functionalize these structures. This contribution describes our efforts in the synthesis of pyridinium salts $\mathbf{1}$ bearing free tertiary amine functionalities, which could serve as mediators in hydride transfer reactions. 


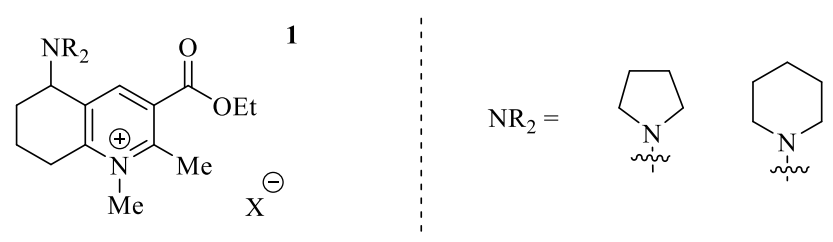

\section{Results and Discussion}

The synthetic route designed for this purpose (Scheme 1) can be divided into two parts: first, benzylic amines 4 are prepared in a five step sequence starting from commercially available and inexpensive ethyl 3-oxobutanoate, ammonium carbamate and cyclohexane-1,3-dione, following well-established procedures. The second and more challenging part requires protection of the amino group in order to neutralize its nucleophilicity, which is necessary to achieve selective $N$ methylation of the pyridine ring. As a straightforward solution for the selective protection of the tertiary amino group, a proton was chosen as protecting group, which is easily introduced and removed. Protonation with one equivalent of acid should lead to the ammonium salt $\mathbf{3}$, leaving the less basic pyridine $\mathrm{N}$ atom free. Therefore, $\mathbf{3}$ should react selectively at the pyridine $\mathrm{N}$ atom with a methylating agent. Subsequent deprotonation with a suitable base should then provide the desired functionalized pyridinium salt 1 with a free amine function.

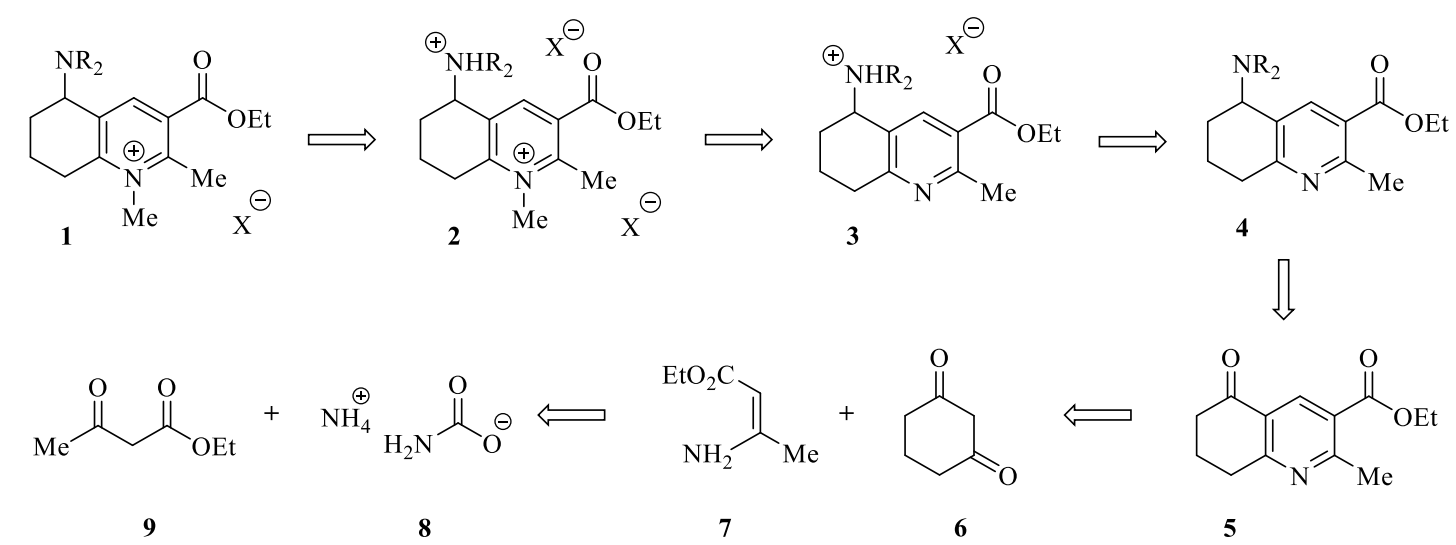

Scheme 1. Retrosynthesis of pyridinium salts 1.

\section{Synthesis of aminopyridines}

Ketone 5 was easily prepared in two steps from commercially available compounds following established procedures (Scheme 2): ${ }^{.5}$ first, treatment of ethyl acetoacetate 9 with one equivalent of ammonium carbamate $\mathbf{8}$ in $\mathrm{MeOH}$ gave (Z)-ethyl 3-aminobut-2-enoate 7 in quantitative yield. ${ }^{5}$ Subsequent condensation of 7 with 1,3-cyclohexanedione and ethyl orthoformate in refluxing acetic acid led to the desired ketone 5 in moderate but acceptable yield (54\%). ${ }^{6}$ 
<smiles>CCOC(=O)CC(C)=O</smiles>

9

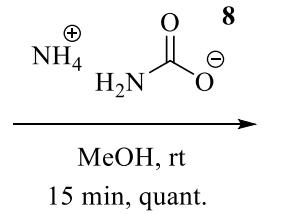

15 min, quant.

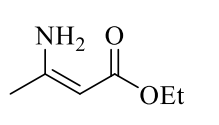<smiles>O=C1CCCC(=O)C1</smiles>

6

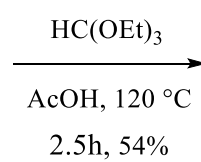<smiles>CCOC(=O)c1cc2c(nc1C)CCCC2=O</smiles>

Scheme 2. Synthesis of ketone 5

Attempts to prepare amine $\mathbf{4}$ either by reductive amination of ketone $\mathbf{5}$ or by Mitsunobu reaction of the corresponding alcohol $\mathbf{1 0}$ resulted in very low yields due to substantial formation of side products and decomposition. Therefore, a three step procedure involving reduction of ketone 5 to alcohol 11 followed by formation of the desired amine via treatment of $\mathbf{1 0}$ with $\mathrm{MsCl}$ and subsequent nucleophilic displacement with a secondary amine was evaluated. Reduction of ketone 5 with $\mathrm{NaBH}_{4}$ in $\mathrm{MeOH}$ gave the alcohol 10 in quantitative yield (Scheme 3). Subsequent treatment with $\mathrm{MsCl}$ in the presence of triethylamine led to a 4:1 mixture of chloride $\mathbf{1 1}$ and alkene 12, which were easily separated by column chromatography.<smiles>Cc1nc2c(cc1C(=O)O)C(=O)CCC2</smiles>

5

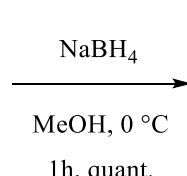

1h, quant.

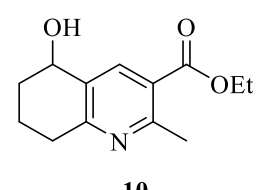

10

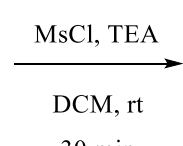

$30 \mathrm{~min}$

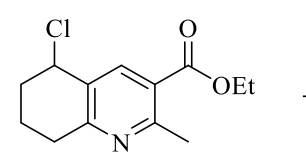

$\mathbf{1 1}, 81 \%$<smiles>CCOC(=O)c1cc2c(nc1C)CCC=C2</smiles>

12

Scheme 3. Conversion of ketone 5 to chloride 11.

We were pleased to find that chloride $\mathbf{1 1}$ reacted readily with a variety of secondary amines in refluxing acetonitrile ${ }^{7}$ to afford the desired amino pyridines in good to excellent yields (Table 1). The best results were obtained with cyclic secondary amines (entries 2-5). The reaction with L-prolinol (entry 5) generated a 1:1 mixture of two diastereoisomers, which could be separated by column chromatography. Amino pyridines 14-17 were readily accessible in gram quantities by this convenient synthetic procedure. After purification by column chromatography these products proved to be very stable and easy to handle solids. In contrast to cyclic amines, diethylamine (entry 1) proved to be unreactive, and even after longer reaction times the conversion was still very low. 
Table 1. Reaction of chloride $\mathbf{1 1}$ with secondary amines
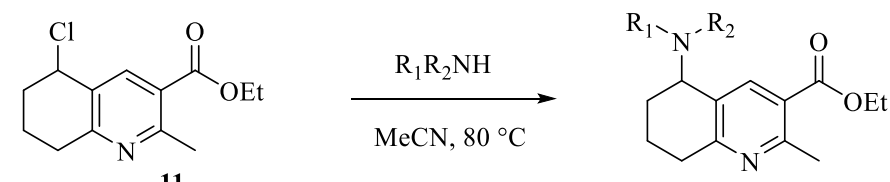

11

\begin{tabular}{ccccc}
\hline Entry & Product & Time $[\mathrm{h}]$ & $\begin{array}{c}\text { Yield } \\
{[\%]^{\mathrm{a}}}\end{array}$ \\
\hline 1 & $\mathbf{1 3}$ & 48 & $<5^{\mathrm{b}}$ \\
2 & $\mathbf{1 4}$ & 24 & 98 \\
5 & $\mathbf{1 5}$ & 20 & 80 \\
\hline
\end{tabular}

${ }^{a}$ Pure isolated product after chromatography. ${ }^{b}$ Estimated by ${ }^{1} \mathrm{H}$ NMR analysis of the crude reaction mixture. ${ }^{c}$ Combined yield of both diastereoisomers.

\section{Synthesis of pyridinium salts}

Preliminary experiments showed that, as expected, direct methylation of amine $\mathbf{1 4}$ led to inseparable mixtures of $\mathbf{1 8}$, in which the tertiary amino group was methylated, and 19, in which both nitrogen atoms were methylated, along with variable amounts of unreacted starting material (Table 2).

Obviously the amine functionality had to be protected for the preparation of the desired pyridinium salts. As outlined in Scheme 1, we thought that selective protonation of the tertiary amine would provide an effective way to achieve selective $N$-methylation of the pyridine ring. ${ }^{8}$ To examine whether selective monoprotonation was indeed possible and whether the protonated amines were sufficiently stable under methylation conditions, we studied the protonation of amine 14 with various Brønsted acids (Table 3). 
Table 2. Direct methylation of amine 14

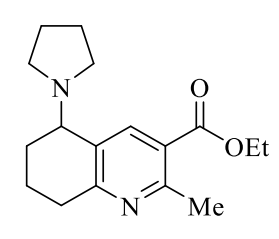

14

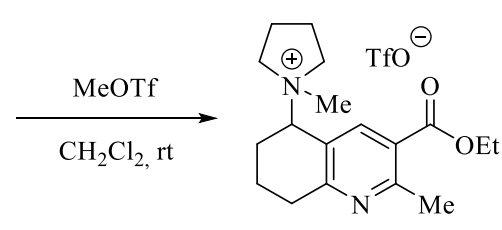

18

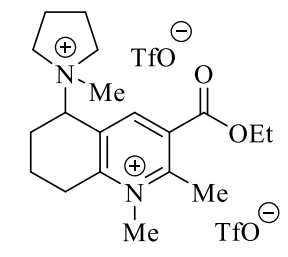

19

\begin{tabular}{cccc}
\hline Entry & $\begin{array}{r}\text { MeOTf } \\
\text { [equiv.] }\end{array}$ & product & ratio $^{\mathrm{a}}$ \\
\hline 1 & 1.1 & $\mathbf{1 8}+\mathbf{1 9}$ & $1: 1$ \\
2 & 0.9 & $\mathbf{1 8}+\mathbf{1 9}$ & $2: 1$ \\
\hline
\end{tabular}

${ }^{\text {a }}$ Estimated by ${ }^{1} \mathrm{H}$ NMR analysis of the crude reaction mixture.

Table 3. Protonation of amine $\mathbf{1 4}$

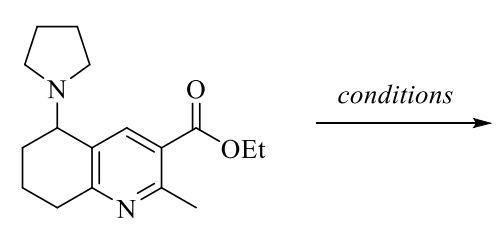

14

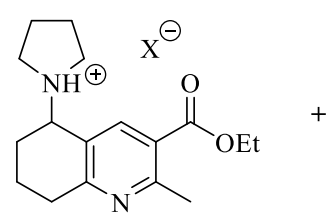

$20\left(\mathrm{X}=\mathrm{TfO}^{-}\right)$

$22\left(\mathrm{X}=\mathrm{TosO}^{-}\right)$

$24\left(\mathrm{X}=\mathrm{BF}_{4}^{-}\right)$

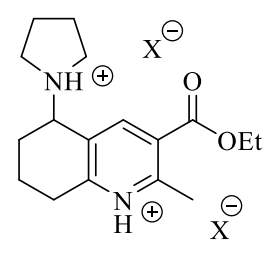

$21\left(\mathrm{X}=\mathrm{TfO}^{-}\right)$

$23\left(\mathrm{X}=\mathrm{Tos}^{-}\right)$ $25\left(\mathrm{X}=\mathrm{BF}_{4}^{-}\right)$

\begin{tabular}{ccccccc}
\hline Entry & Acid & Equiv. & Solvent & $\begin{array}{c}\text { Time } \\
(\mathrm{min})\end{array}$ & Product & $\begin{array}{c}\text { Yield }^{\mathrm{a}} \\
(\%)\end{array}$ \\
\hline 1 & $\mathrm{HClEt}{ }_{2} \mathrm{O}$ & 1.0 & $\mathrm{Et}_{2} \mathrm{O}$ & 10 & - & - \\
\hline 2 & & 0.9 & $\mathrm{DCM}$ & 20 & $\mathbf{2 0}$ & 77 \\
3 & $\mathrm{TfOH}$ & 2.0 & $\mathrm{DCM}$ & 20 & $\mathbf{2 1}$ & 82 \\
\hline 4 & & 0.9 & $\mathrm{DCM}$ & 20 & $\mathbf{2 2}$ & 84 \\
5 & $\mathrm{TosOH}$ & 2.0 & $\mathrm{DCM}^{2}$ & 20 & $\mathbf{2 3}$ & 90 \\
\hline 6 & & 0.9 & $\mathrm{Et}_{2} \mathrm{O}$ & 10 & $\mathbf{2 4}$ & 95 \\
7 & $\mathrm{HBF}_{4}$ & 2.0 & $\mathrm{Et}_{2} \mathrm{O}$ & 10 & $\mathbf{2 4}$ & 95 \\
\hline
\end{tabular}

${ }^{a}$ Estimated by ${ }^{1} \mathrm{H}$ NMR analysis of the crude reaction mixture. 
Initial experiments using $\mathrm{HCl} \mathrm{Et}_{2} \mathrm{O}$ failed, resulting in rapid decomposition of the starting amine (entry 1). Protonation with trifluoromethanesulfonic acid (entries 2 and 3) yielded the desired compound 20, but also led to the formation of unidentified side products. Protonation with 0.9 or 2.0 equivalents of $p$-toluenesulfonic acid afforded the mono- and diprotonated compounds $\mathbf{2 2}$ and 23, respectively, in good yields (entries 4 and 5). Unfortunately, the resulting trifluoromethanesulfonate and $p$-toluenesulfonate salts proved to be highly sensitive to air and difficult to handle. Eventually, tetrafluoroboric acid diethyl etherate proved to be the optimal choice, affording 24 in $95 \%$ yield (entry 6). The resulting salt was found to be stable towards air and moisture, and the corresponding bisprotonated species was not detected even when two equivalents of acid were added (entry 7). In an analogous manner, the monoprotonated compounds 26 and 27 were cleanly obtained in $97 \%$ yield (Scheme 4).
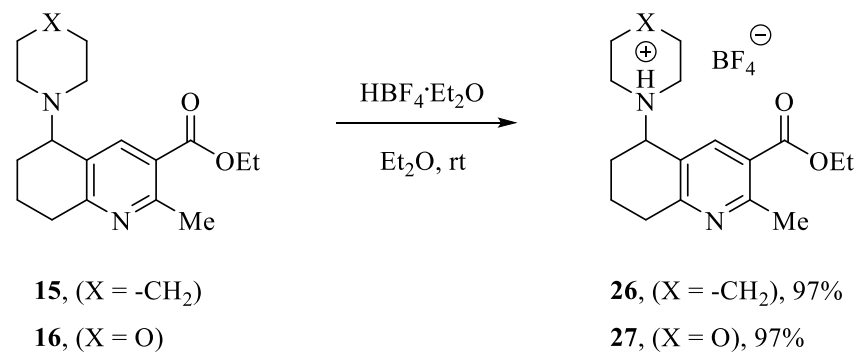

Scheme 4. Protonation of $\mathbf{1 5}$ and $\mathbf{1 6 .}$

\section{Methylation}

With reliable procedures for the preparation of monoprotonated amino pyridines in hand, we studied the reaction of standard methylating agents such as methyl trifluoromethanesulfonate, trimethyloxonium tetrafluoroborate $\left(\mathrm{Me}_{3} \mathrm{OBF}_{4}\right)$, dimethyl sulfate or methyl tosylate with ammonium salt 24. While treatment with $\mathrm{MeOTos}$ and $\mathrm{Me}_{2} \mathrm{SO}_{4}$ led to the formation of complex mixtures, the use of $\mathrm{Me}_{3} \mathrm{OBF}_{4}$ resulted in formation of the desired biscationic salt, albeit with low conversion. MeOTf gave more promising results and, therefore, further studies focused on this reagent. After some experimentation, we identified dioxane as ideal solvent for this transformation (Table 4).

Variation of the temperature revealed that an increase from $\mathrm{rt}$ to $50^{\circ} \mathrm{C}$ had a negative impact on conversion (entries 1 and 2). Because of the relatively low solubility of $\mathbf{2 4}$ in dioxane, solvent mixtures of dioxane and $10 \% \mathrm{DMF}$ were tested to enhance solubility, but the reaction was slower in this case (entry 3). Notably, shorter reaction times led to higher conversion (entries 4-6), which is consistent with the observation that the resulting biscationic salts are unstable and probably decompose over extended reaction times. Lowering the concentration from $0.2 \mathrm{M}$ to 0.1 $\mathrm{M}$ increased the conversion from $22 \%$ to $47 \%$ (entry 6), a trend that became even more obvious when the concentration was lowered to $0.02 \mathrm{M}$, resulting in an improved conversion of $70 \%$ (entry 8). As observed before higher temperatures led to lower yields (entry 9). At $0{ }^{\circ} \mathrm{C}$, the ${ }^{1} \mathrm{H}$ NMR spectra showed no remaining starting material after $1 \mathrm{~h}$, but the formation of almost 
$20 \%$ of an unidentified side product was observed (entry 10). The use of an excess of MeOTf had a negative effect on conversion (entries 11 and 12). In summary, stirring a $0.02 \mathrm{M}$ solution of $\mathbf{2 4}$ in dioxane at room temperature for 4 hours proved to be optimal for this reaction (entry 8). Under these conditions, conversions to the biscationic salt in the range of $70 \%$ were consistently obtained. Using the same procedure compound $\mathbf{2 6}$ was formed with $60 \%$ conversion (Scheme 5).

Table 4. Methylation with methyl trifluoromethanesulfonate in dioxane

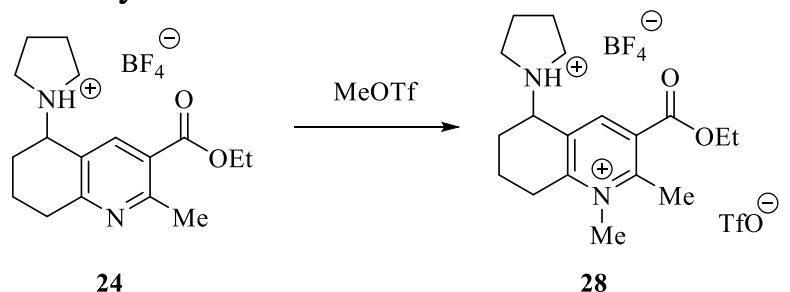

\begin{tabular}{|c|c|c|c|c|c|c|}
\hline Entry & $\begin{array}{l}\text { MeOTf } \\
\text { (equiv.) }\end{array}$ & Solvent & $\begin{array}{c}c \\
{[\mathrm{~mol} / \mathrm{L}]}\end{array}$ & $\begin{array}{c}\text { Time } \\
{[\mathrm{h}]}\end{array}$ & $\mathrm{T}\left[{ }^{\circ} \mathrm{C}\right]$ & $\begin{array}{c}\text { Conv. }^{a} \\
{[\%]}\end{array}$ \\
\hline 1 & 1 & dioxane & 0.2 & 18 & $\mathrm{rt}$ & 22 \\
\hline 2 & 1 & dioxane & 0.2 & 18 & 50 & 12 \\
\hline 3 & 1 & $\begin{array}{l}\text { dioxane }+ \\
10 \% \mathrm{DMF}\end{array}$ & 0.2 & 18 & $\mathrm{rt}$ & 19 \\
\hline 4 & 1 & dioxane & 0.1 & 18 & $\mathrm{rt}$ & 33 \\
\hline 5 & 1 & dioxane & 0.1 & 1.5 & $\mathrm{rt}$ & 38 \\
\hline 6 & 1 & dioxane & 0.1 & 4 & $\mathrm{rt}$ & 47 \\
\hline 7 & 1 & dioxane & 0.05 & 4 & $\mathrm{rt}$ & 56 \\
\hline 8 & 1 & dioxane & 0.02 & 4 & $\mathrm{rt}$ & 70 \\
\hline 9 & 1 & dioxane & 0.02 & 4 & 50 & 29 \\
\hline 10 & 1 & dioxane & 0.05 & 4 & $0-15$ & 100 \\
\hline 11 & 2 & dioxane & 0.05 & 4 & $\mathrm{rt}$ & 25 \\
\hline 12 & 4 & dioxane & 0.2 & 4 & $\mathrm{rt}$ & 29 \\
\hline
\end{tabular}

${ }^{\text {a }}$ Estimated by ${ }^{1} \mathrm{H}$ NMR analysis of the crude reaction mixtures. 


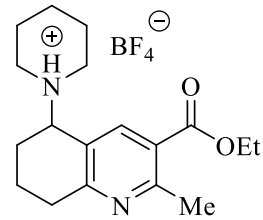

26

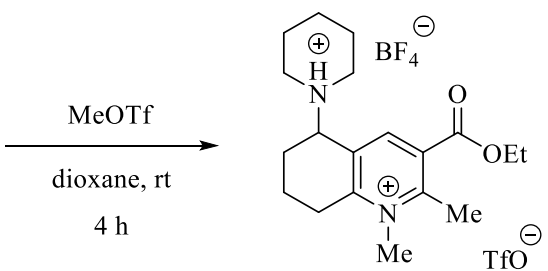

29

Scheme 5. Methylation of the protonated amino pyridine 26.

\section{Preparation of $\boldsymbol{N}$-methyl pyridium salts with a free amino function}

In order to complete the synthesis of amino pyridinium salts we needed a suitable base for the deprotonation of the amonium salts that allowed easy removal of the resulting protonated base. We reasoned that sodium hydride would be ideal because the protonated base in this case is hydrogen gas. After methylation of pyridine 24, addition of 1.5 equivalents of sodium hydride to the reaction mixture in dioxane yielded the pyridinium salt $\mathbf{3 0}$ and minor amounts of the unmethylated aminopyridine 14 (Scheme 6). Due to the higher solubility of $\mathbf{1 4}$, it was possible to separate the two compounds simply by washing with pentane. The generated $\mathrm{NaBF}_{4}$ could then be removed by dissolving $\mathbf{3 0}$ in dichloromethane and subsequent filtration through an HPLC filter. In this way the desired product 30 was obtained in $64 \%$ yield. This procedure was also successfully applied to the synthesis of pyridinium salt 31, which was isolated in $57 \%$ yield.
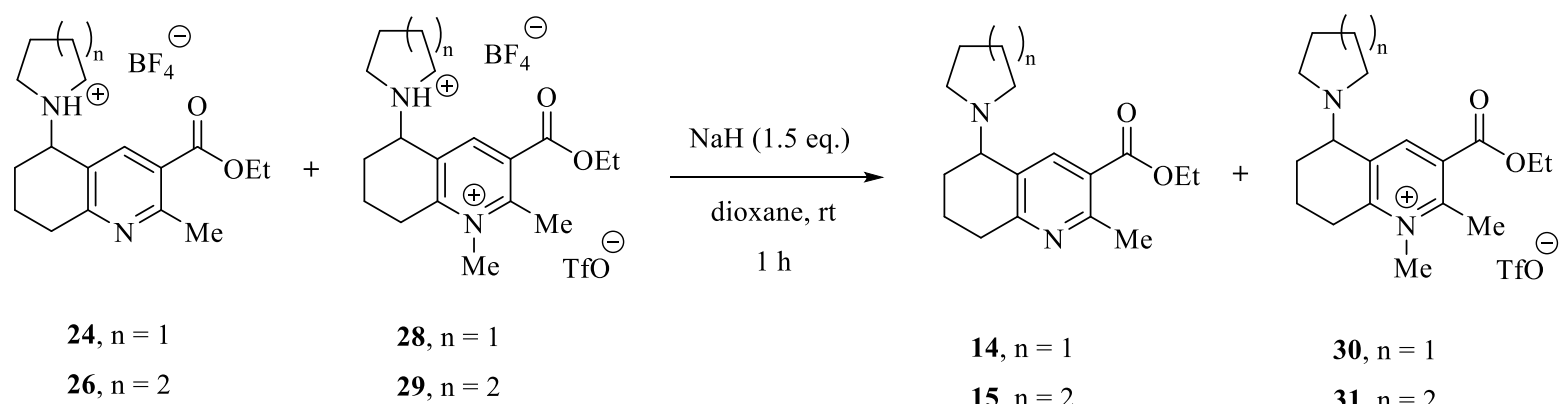

$$
\begin{aligned}
& 14, n=1 \\
& 15, n=2
\end{aligned}
$$$$
\text { 30, } \mathrm{n}=1
$$$$
\text { 31, } \mathrm{n}=2
$$

Scheme 6. Deprotonation of 28 and 29.

\section{Conclusions}

An efficient straightforward synthetic route to $N$-methylpyridinium salts containing a tertiary amine function has been developed. Selective $N$-methylation of the pyridine ring, which is the key step of the synthesis, has been achieved by protonation of the amino group and subsequent reaction with methyl triflate, followed by deprotonation with sodium hydride. Through this procedure amino-functionalized pyridinium salts become conveniently accessible in high purity. 


\section{Experimental Section}

General. All chemicals were used as received from the chemical supplier without further purification unless otherwise noted. DCM, $n$-pentane and $\mathrm{Et}_{2} \mathrm{O}$ were purified with a Grubbs type purification column system (PureSolv, Innovative Technology Inc.). All other solvents were reagent grade quality (Sigma-Aldrich over mol. sieves sealed with crown cap).

NMR Spectroscopy: spectra were measured on a Bruker Avance $400(400 \mathrm{MHz})$ or Bruker Avance $500(500 \mathrm{MHz}) \mathrm{DRX}$ spectrometer. Chemical shifts $\delta$ are given in ppm and referenced to the residual solvent peaks: $\mathrm{CDCl}_{3}(\delta 7.26)$, DMSO $(\delta 2.50),{ }^{13} \mathrm{C}-\mathrm{NMR}: \mathrm{CDCl}_{3}(\delta 77.16)$, DMSO $(\delta 39.52)$. Assignment of ${ }^{1} \mathrm{H}$ and ${ }^{13} \mathrm{C}$ signals was partly made by $2 \mathrm{D}-\mathrm{NMR}$, namely COSY, HMQC, HMBC and NOESY. ${ }^{13} \mathrm{C}$ spectra were recorded in ${ }^{1} \mathrm{H}$ decoupled mode. Signal multiplicity: s (singlet), d (doublet), $\mathrm{t}$ (triplet), q (quartet), quint (quintet), sext (sextet), $\mathrm{m}$ (multiplet). The notation br stands for broad.

Mass spectra were measured on a VG70-250 (electron ionization (EI)) mass spectrometer or a MAR 312 (fast atom bombardment (FAB)) mass spectrometer. FAB was performed with 3nitrobenzyl alcohol (NBA) as matrix. ESI MS spectra were measured on a Finnigan MAT LCQ and a on a Varian 1200L triple Quad MS/MS. The signals are given in mass to charge ratio $(\mathrm{m} / \mathrm{z})$. The fragment and intensities are given in brackets. All values are rounded to the nearest whole number.

Infrared spectra were measured on a Perkin Elmer 1600 series FTIR spectrometer. Solid samples were measured as $\mathrm{KBr}$ discs or as thin films on $\mathrm{NaCl}$ plates. Absorption bands are given in wave numbers $v_{\max }\left(\mathrm{cm}^{-1}\right)$. The peak intensity is assigned with $\mathrm{s}$ (strong), $\mathrm{m}$ (medium) and $\mathrm{w}$ (weak). The index br stands for broad.

Melting points were determined on a Büchi 535 melting point apparatus. Optical Rotations $\left([\alpha]_{\mathrm{D}}^{20}\right)$ were measured on a Perkin Elmer Polarimeter 341 in a $1 \mathrm{dm}$ cuvette at $20^{\circ} \mathrm{C}$. The concentration (c) is given in $\mathrm{g} / 100 \mathrm{~mL}$. Thin Layer Chromatography: TLC plates were obtained from Macherey-Nagel (Polygram SIL G/UV 254 and Polygram Alox N/UV $254,0.2 \mathrm{~mm}$ silica with fluorescence indicator, $40 \times 80 \mathrm{~mm})$. For visualization UV light $(254 \mathrm{~nm}, 366 \mathrm{~nm})$ or with basic permanganate solution or ceric ammonium molybdate solution. Elemental analyses were measured on a Leco CHN-900 analyzer.

Ethyl (Z)-3-aminobut-2-enoate (7). To a solution of ethyl acetoacetate $9(19.4 \mathrm{~mL}, 154 \mathrm{mmol})$ in methanol $(160 \mathrm{~mL})$ ammonium carbamate $8(11.7 \mathrm{~g}, 154 \mathrm{mmol})$ was added in one portion. The resulting suspension was stirred at room temperature for $1.5 \mathrm{~h}$. During that time all solid material dissolved to give a clear yellow solution. The reaction mixture was concentrated to dryness yielding title compound 7 (19.8 g, $153 \mathrm{mmol}$, quant.) as yellow liquid. ${ }^{1} \mathrm{H}-\mathrm{NMR}$ (400 $\left.\mathrm{MHz}, \mathrm{CDCl}_{3}\right), \delta(\mathrm{ppm}): 7.62$ (b, 2H, NH$\left.H_{2}\right), 4.30(\mathrm{~s}, 1 \mathrm{H}, \mathrm{CH}), 3.90\left(\mathrm{q}, J_{H H} 7.1 \mathrm{~Hz}, 2 \mathrm{H}, \mathrm{CH}_{2} \mathrm{CH}_{3}\right.$ ), $1.71\left(\mathrm{~s}, 3 \mathrm{H}, \mathrm{CH}_{3}\right), 1.06\left(\mathrm{t}, J_{H H} 7.1 \mathrm{~Hz}, 3 \mathrm{H}, \mathrm{CH}_{2} \mathrm{CH}_{3}\right) ;{ }^{13} \mathrm{C}\left\{{ }^{1} \mathrm{H}\right\}-\mathrm{NMR}\left(101 \mathrm{MHz}, \mathrm{CDCl}_{3}\right), \delta$ (ppm): 170.5 (s, $C=\mathrm{O}), 160.6$ (s, $\left.C-\mathrm{NH}_{2}\right), 83.7$ (s, $\left.C \mathrm{H}\right), 58.6$ (s, $\left.C \mathrm{H}_{2} \mathrm{CH}_{3}\right), 22.2\left(\mathrm{~s}, C \mathrm{H}_{3}\right), 14.8$ (s, $\left.\mathrm{CH}_{2} \mathrm{CH}_{3}\right)$; TLC: $\mathrm{R}_{f} 0.36\left(\mathrm{SiO}_{2}\right.$, cyclohexane/EtOAc 1:1). 
Ethyl 2-methyl-5-oxo-5,6,7,8-tetrahydroquinoline-3-carboxylate (5). A solution of 1,3cyclohexanedione $(17.3 \mathrm{~g}, 153 \mathrm{mmol})$, triethyl orthoformate $(90 \mathrm{~mL}, 540 \mathrm{mmol})$ and (Z)-ethyl 3aminobut-2-enoate 7 (19.8 g, $153 \mathrm{mmol})$ in acetic acid $(13 \mathrm{~mL})$ was refluxed for $2.5 \mathrm{~h}$ under an argon atmosphere. Volatile compounds were removed in vacuo. The resulting residue was dissolved in EtOAc $(200 \mathrm{~mL})$ and washed with saturated $\mathrm{Na}_{2} \mathrm{CO}_{3}$ solution $(150 \mathrm{~mL})$ and brine $(50 \mathrm{~mL})$. The organic layers were concentrated to obtain crude product as red solid. Purification by column chromatography $\left(\mathrm{SiO}_{2}\right.$, cyclohexane/EtOAc $\left.3: 1,7 \times 25 \mathrm{~cm}\right)$ afforded the desired product 5 (19.5 g, $83.6 \mathrm{mmol}, 54 \%)$ as an orange solid. Mp: 86-88 ${ }^{\circ} \mathrm{C}$; ${ }^{1} \mathrm{H}-\mathrm{NMR}(400 \mathrm{MHz}$, $\left.\mathrm{CDCl}_{3}\right), \delta(\mathrm{ppm}): 8.70(\mathrm{~s}, 1 \mathrm{H}, \mathrm{Ar}-H), 4.36\left(\mathrm{q}, J_{\mathrm{HH}} 7.1 \mathrm{~Hz}, 2 \mathrm{H}, \mathrm{CH}_{2} \mathrm{CH}_{3}\right), 3.11$ (t, $J_{H H} 6.2 \mathrm{~Hz}, 2 \mathrm{H}$, $\left.\mathrm{CH}_{2} \mathrm{CH}_{3}\right), 2.84\left(\mathrm{~s}, 3 \mathrm{H}, \mathrm{CH}_{3}\right), 2.70-2.63\left(\mathrm{~m}, 2 \mathrm{H}, \mathrm{CH}_{2}\right), 2.21-2.14\left(\mathrm{~m}, 2 \mathrm{H}, \mathrm{CH}_{2}\right), 1.38$ (t, JHH 7.1 $\left.\mathrm{Hz}, 3 \mathrm{H}, \mathrm{CH}_{2} \mathrm{CH}_{3}\right) ;{ }^{13} \mathrm{C}\left\{{ }^{1} \mathrm{H}\right\}-\mathrm{NMR}\left(101 \mathrm{MHz}, \mathrm{CDCl}_{3}\right), \delta(\mathrm{ppm}): 197.5$ (s, $\left.C=\mathrm{O}\right), 166.2$ (s, $\left.C=\mathrm{O}\right)$, 166.0 (s, Ar-C), 164.6 (s, Ar-C), 137.8 (s, Ar-CH), 126.2 (s, Ar-C), 125.0 (s, Ar-C), 61.8 (s, $\left.\mathrm{CH}_{2} \mathrm{CH}_{3}\right), 38.9\left(\mathrm{~s}, \mathrm{CH}_{2}\right), 33.0\left(\mathrm{~s}, \mathrm{CH}_{2}\right), 25.6\left(\mathrm{~s}, \mathrm{CH}_{2}\right), 22.0\left(\mathrm{~s}, \mathrm{CH}_{3}\right), 14.7\left(\mathrm{~s}, \mathrm{CH}_{2} \mathrm{CH}_{3}\right)$; IR (neat): $v_{\max }\left(\mathrm{cm}^{-1}\right) 2955 \mathrm{w}, 1721 \mathrm{~s}, 1684 \mathrm{~s}, 1586 \mathrm{~m}, 1553 \mathrm{~m}, 1434 \mathrm{~m}, 1368 \mathrm{~m}, 1244 \mathrm{~s}, 1212 \mathrm{~s}, 1179 \mathrm{~s}, 1021 \mathrm{~m}$, 909w, 780m, 670w; EA $\left(\mathrm{C}_{13} \mathrm{H}_{15} \mathrm{NO}_{3}\right)$ : calc.: C 66.94, H 6.48, N 6.00; found: C 66.49, H 6.78, N 6.10; MS (EI, $70 \mathrm{eV}, 150{ }^{\circ} \mathrm{C}$ ) $\mathrm{m} / z$ (\%): 233 (100) [M] ${ }^{+}, 205$ (99), 188 (72), 177 (63), 132 (14); TLC: $\mathrm{R}_{f} 0.25\left(\mathrm{SiO}_{2}\right.$, cyclohexane/EtOAc 3:1.

Ethyl 2-methyl-5-hydroxy-5,6,7,8-tetrahydroquinoline-3-carboxylate (10). Ketone 5 (8.10 g, $34.7 \mathrm{mmol})$ was dissolved in methanol $(140 \mathrm{~mL})$ and sodium borohydride $(1.37 \mathrm{~g}, 34.7 \mathrm{mmol})$ was added at $0{ }^{\circ} \mathrm{C}$. The mixture was stirred for $1 \mathrm{~h}$ at $\mathrm{rt}$. The obtained yellow solution was quenched with cold water $(100 \mathrm{~mL})$ and extracted with $\mathrm{DCM}(3 \times 50 \mathrm{~mL})$. The combined organic layers were dried over $\mathrm{MgSO}_{4}$ and filtered. Evaporation of the solvent gave the alcohol 10 (8.20 g, 34.9 mmol, quant.) as a yellow solid. Mp 70-72 ${ }^{\circ} \mathrm{C} ;{ }^{1} \mathrm{H}-\mathrm{NMR}\left(400 \mathrm{MHz}, \mathrm{CDCl}_{3}\right), \delta$ (ppm): $8.25(\mathrm{~s}, 1 \mathrm{H}, \mathrm{Ar}-H), 4.81\left(\mathrm{~s}, 1 \mathrm{H}, \mathrm{CHCH}_{3}\right), 4.35$ (q, $\left.J_{H H} 7.1 \mathrm{~Hz}, 2 \mathrm{H}, \mathrm{CH}_{2} \mathrm{CH}_{3}\right), 3.00-2.79$ $\left(\mathrm{m}, 2 \mathrm{H}, \mathrm{CH}_{2}\right), 2.77\left(\mathrm{~s}, 3 \mathrm{H}, \mathrm{CH}_{3}\right), 2.31(\mathrm{~s}, 1 \mathrm{H}, \mathrm{OH}), 2.11-1.99\left(\mathrm{~m}, 2 \mathrm{H}, \mathrm{CH}_{2}\right), 1.90-1.79(\mathrm{~m}, 2 \mathrm{H}$, $\left.\mathrm{CH}_{2}\right), 1.38\left(\mathrm{t}, J_{H H} 7.1 \mathrm{~Hz}, 3 \mathrm{H}, \mathrm{CH}_{2} \mathrm{CH}_{3}\right) ;{ }^{13} \mathrm{C}\left\{{ }^{1} \mathrm{H}\right\}-\mathrm{NMR}\left(101 \mathrm{MHz}, \mathrm{CDCl}_{3}\right), \delta$ (ppm): $167.0(\mathrm{~s}$, $C=\mathrm{O}), 160.2$ (s, $\operatorname{Ar}-C$ ), 158.5 (s, Ar- $C$ ), 139.3 (s, Ar- $C \mathrm{H}$ ), 132.5 (s, Ar- $C$ ), 123.8 (s, Ar- $C$ ), 67.4 $(\mathrm{s}, \mathrm{CH}), 61.5\left(\mathrm{~s}, \mathrm{CH}_{2} \mathrm{CH}_{3}\right), 32.6\left(\mathrm{~s}, \mathrm{CH}_{2}\right), 32.4\left(\mathrm{~s}, \mathrm{CH}_{2}\right), 24.7\left(\mathrm{~s}, \mathrm{CH}_{2}\right), 19.0\left(\mathrm{~s}, \mathrm{CH}_{3}\right), 14.6(\mathrm{~s}$, $\mathrm{CH}_{2} \mathrm{CH}_{3}$ ); MS (EI, $\left.70 \mathrm{eV}, 150{ }^{\circ} \mathrm{C}\right) \mathrm{m} / \mathrm{z}(\%): 235$ (43) $[\mathrm{M}]^{+}, 220$ (26), 206(100) [M - $\left.\mathrm{CH}_{2} \mathrm{CH}_{3}\right]^{+}$, 190 (37), 179 (59), 162 (34), 151 (13), 144 (12), 77 (12), 65 (11); IR (neat): $v_{\max }\left(\mathrm{cm}^{-1}\right) 3217 \mathrm{w}$, $2932 \mathrm{w}, 1721 \mathrm{~s}, 1564 \mathrm{~m}, 1447 \mathrm{~m}, 1322 \mathrm{~m}, 1257 \mathrm{~s}, 1156 \mathrm{~s}, 1072 \mathrm{~s}, 1021 \mathrm{~m}, 956 \mathrm{w} ;$ TLC: $\mathrm{R}_{f} 0.24\left(\mathrm{SiO}_{2}\right.$, cyclohexane/EtOAc 2:1).

Ethyl 5-chloro-2-methyl-5,6,7,8-tetrahydroquinoline-3-carboxylate (11). To a solution of compound $10(8.20 \mathrm{~g}, 34.9 \mathrm{mmol})$ in DCM $(180 \mathrm{~mL})$ and triethylamine $(7.4 \mathrm{~mL}, 52.3 \mathrm{mmol})$ was added methanesulfonyl chloride $\left(3.3 \mathrm{~mL}, 41.8 \mathrm{mmol}, 1.2\right.$ equiv.) at $0{ }^{\circ} \mathrm{C}$. The mixture was stirred at $\mathrm{rt}$ for $30 \mathrm{~min}$. The resulting solution was extracted with $\mathrm{H}_{2} \mathrm{O}(3 \times 50 \mathrm{~mL})$. The aqueous layers were back extracted each time with DCM $(2 \times 20 \mathrm{~mL})$. The combined organic layers were dried over $\mathrm{MgSO}_{4}$, filtered and concentrated. Purification by column chromatography $\left(\mathrm{SiO}_{2}\right.$, cyclohexane/EtOAc 7:1, $7 \times 25 \mathrm{~cm})$ afforded the desired product $11(6.15 \mathrm{~g}, 24.8 \mathrm{mmol}, 81 \%)$ as an orange oil. ${ }^{1} \mathrm{H}-\mathrm{NMR}\left(400 \mathrm{MHz}, \mathrm{CDCl}_{3}\right), \delta(\mathrm{ppm}): 8.18(\mathrm{~s}, 1 \mathrm{H}, \mathrm{Ar}-H), 5.26(\mathrm{~m}, 1 \mathrm{H}, \mathrm{CH}), 4.37$ 
(q, $\left.J_{H H} 7.2 \mathrm{~Hz}, 2 \mathrm{H}, \mathrm{CH}_{2} \mathrm{CH}_{3}\right), 3.09-2.98\left(\mathrm{~m}, 1 \mathrm{H}, \mathrm{CH}_{2}\right), 2.92-2.80\left(\mathrm{~m}, 1 \mathrm{H}, \mathrm{CH}_{2}\right), 2.78(\mathrm{~s}, 3 \mathrm{H}$, $\left.\mathrm{CH}_{3}\right), 2.32-2.13\left(\mathrm{~m}, 3 \mathrm{H}, 2 \times \mathrm{CH}_{2}\right), 1.99-1.90\left(\mathrm{~m}, 1 \mathrm{H}, \mathrm{CH}_{2}\right), 1.39$ (t, $\left.J_{H H} 7.1 \mathrm{~Hz}, 3 \mathrm{H}, \mathrm{CH}_{2} \mathrm{CH}_{3}\right)$; ${ }^{13} \mathrm{C}\left\{{ }^{1} \mathrm{H}\right\}-\mathrm{NMR}\left(101 \mathrm{MHz}, \mathrm{CDCl}_{3}\right), \delta(\mathrm{ppm}): 166.6$ (s, C=O), 159.8 (s, Ar-C), 159.6 (s, Ar$C), 140.7(\mathrm{~s}, \mathrm{Ar}-\mathrm{CH}), 129.9(\mathrm{~s}, \mathrm{Ar}-\mathrm{C}), 124.0(\mathrm{~s}, \mathrm{Ar}-\mathrm{C}), 61.6(\mathrm{~s}, \mathrm{CH}), 57.1\left(\mathrm{~s}, \mathrm{CH}_{2} \mathrm{CH}_{3}\right), 32.8(\mathrm{~s}$, $\left.\mathrm{CH}_{2}\right), 32.5$ (s, $\left.\mathrm{CH}_{2}\right), 25.1$ (s, $\left.\mathrm{CH}_{2}\right), 18.6\left(\mathrm{~s}, \mathrm{CH}_{3}\right), 14.7\left(\mathrm{~s}, \mathrm{CH}_{2} \mathrm{CH}_{3}\right)$; EA $\left(\mathrm{C}_{13} \mathrm{H}_{16} \mathrm{ClNO}_{2}\right)$ : calc.: $\mathrm{C}$ 61.54, H 6.36, N 5.52; found: C 61.37, H 6.47, N 5.50; MS (EI, 70 eV, $50{ }^{\circ} \mathrm{C}$ ) $\mathrm{m} / z$ (\%): 253 (11) $[\mathrm{M}]^{+}, 218(100)\left[\mathrm{M}-\mathrm{CH}_{2} \mathrm{CH}_{3}\right]^{+}, 190$ (24), 144 (12); IR (neat): $v_{\max }\left(\mathrm{cm}^{-1}\right)$ 2950w, 2358w, 1722s, 1560w, 1445w, 1437w, 1269m, 1241w, 1179w, 1064w; TLC: $\mathrm{R}_{f} 0.55$. ( $\mathrm{SiO}_{2}$, cyclohexane / EtOAc $2: 1)$.

General procedure for formation of amino pyridines. To a solution of 11 (1.0 eq.) in acetonitrile $(0.2 \mathrm{M})$ was added the corresponding amine $\left(2.0\right.$ eq.) at $0{ }^{\circ} \mathrm{C}$. The solution was heated to reflux $\left(80^{\circ} \mathrm{C}\right.$ ) for 24 hours. The reaction mixture was allowed to cool down to room temperature and the mixture was extracted with $\mathrm{H}_{2} \mathrm{O}(30 \mathrm{~mL})$. The aqueous layer was extracted with DCM $(3 \times 20 \mathrm{~mL})$. The combined organic layers were dried over $\mathrm{MgSO}_{4}$, filtered and the solvent was removed under reduced pressure to afford a dark solid.

Ethyl 2-methyl-5-(pyrrolidin-1-yl)-5,6,7,8-tetrahydroquinoline-3-carboxylate (14). The reaction was set up according to the general procedure using $11(2.0 \mathrm{~g}, 6.38 \mathrm{mmol})$ in acetonitrile $(30 \mathrm{~mL})$ and pyrrolidine $(1.05 \mathrm{~mL}, 12.8 \mathrm{mmol})$. Purification by column chromatography $\left(\mathrm{SiO}_{2}\right.$, cyclohexane/EtOAc 3:1 $\rightarrow 1: 2,3 \times 15 \mathrm{~cm})$ yielded the desired product $14(1.80 \mathrm{~g}, 6.24 \mathrm{mmol}$, $98 \%$ ) as a yellow solid. $\mathrm{Mp} 46-48{ }^{\circ} \mathrm{C} ;{ }^{1} \mathrm{H}-\mathrm{NMR}\left(400 \mathrm{MHz}, \mathrm{CDCl}_{3}\right), \delta(\mathrm{ppm}): 8.13$ (s, $\left.1 \mathrm{H}, \mathrm{Ar}-H\right)$, 4.41-4.29 (m, 2H, $\left.\mathrm{CH}_{2} \mathrm{CH}_{3}\right), 3.63-3.58(\mathrm{~m}, 1 \mathrm{H}, \mathrm{CH}), 3.05-2.95\left(\mathrm{~m}, 1 \mathrm{H}, \mathrm{CH}_{2}\right), 2.92-2.80(\mathrm{~m}, 1 \mathrm{H}$, $\left.\mathrm{CH}_{2}\right), 2.76\left(\mathrm{~s}, 3 \mathrm{H}, \mathrm{CH}_{3}\right), 2.66-2.58\left(\mathrm{~m}, 2 \mathrm{H}, \mathrm{CH}_{2}\right), 2.49-2.41\left(\mathrm{~m}, 2 \mathrm{H}, \mathrm{CH}_{2}\right), 2.33-2.13(\mathrm{~m}, 1 \mathrm{H}$, $\mathrm{CH}_{2}$ ), 1.98-1.87 (m, 1H, $\left.\mathrm{CH}_{2}\right), 1.83-1.69\left(\mathrm{~m}, 6 \mathrm{H}, 3 \times \mathrm{CH}_{2}\right), 1.38$ (q, $\left.J_{H H} 7.1 \mathrm{~Hz}, 3 \mathrm{H}, \mathrm{CH}_{2} \mathrm{CH}_{3}\right)$; ${ }^{13} \mathrm{C}\left\{{ }^{1} \mathrm{H}\right\}$-NMR $\left(101 \mathrm{MHz}, \mathrm{CDCl}_{3}\right), \delta(\mathrm{ppm}): 167.4$ (s, $\left.C=\mathrm{O}\right), 161.1$ (s, Ar-C), 157.7 (s, Ar-C), 139.7 (s, Ar- $\mathrm{CH}$ ), 131.8 (s, Ar-C), 122.9 (s, Ar-C), 61.3(s, $\left.\mathrm{CH}_{2} \mathrm{CH}_{3}\right), 59.8(\mathrm{~s}, \mathrm{CH}), 50.2$ (s, 2 $\left.\times \mathrm{CH}_{2}\right), 32.8\left(\mathrm{~s}, C \mathrm{H}_{2}\right), 24.8\left(\mathrm{~s}, \mathrm{CH}_{2}\right), 24.2\left(\mathrm{~s}, C \mathrm{H}_{2}\right), 24.0\left(\mathrm{~s}, 2 \times \mathrm{CH}_{2}\right), 19.3\left(\mathrm{~s}, C \mathrm{H}_{3}\right), \quad 14.7(\mathrm{~s}$, $\left.\mathrm{CH}_{2} \mathrm{CH}_{3}\right)$; EA $\left(\mathrm{C}_{17} \mathrm{H}_{24} \mathrm{~N}_{2} \mathrm{O}_{2}\right)$ : calc.: C 70.80, $\mathrm{H}$ 8.39 N 9.71; found: C 70.79, H 8.27, N 9.64; MS (EI, $\left.70 \mathrm{eV}, 150{ }^{\circ} \mathrm{C}\right) \mathrm{m} / \mathrm{z}(\%): 288(31)\left[\mathrm{M}^{+}, 259\right.$ (35), 245 (13), 231 (29), 217 (100) [M$\left.\mathrm{C}_{4} \mathrm{H}_{9} \mathrm{~N}\right]^{+}, 190$ (26), 172 (20), 144 (28), 70 (18); IR (neat): $v_{\max }\left(\mathrm{cm}^{-1}\right)$ 2978w, 2936w, 2880w, $1719 \mathrm{~m}, 1455 \mathrm{w}, 1316 \mathrm{~s}, 1262 \mathrm{~m}, 1196 \mathrm{~m}, 1140 \mathrm{~s}, 1106 \mathrm{~s}, 1057 \mathrm{~s}, 1005 \mathrm{~s}, 971 \mathrm{~m}, 855 \mathrm{w}, 778 \mathrm{~s}, 724 \mathrm{~m}$; TLC: $\mathrm{R}_{f} 0.15\left(\mathrm{SiO}_{2}\right.$, cyclohexane/EtOAc 3:1).

Ethyl 2-methyl-5-(piperidin-1-yl)-5,6,7,8-tetrahydroquinoline-3-carboxylate (15). The reaction was set up according to the general procedure using $\mathbf{1 1}(2.00 \mathrm{~g}, 6.38 \mathrm{mmol})$ in acetonitrile $(30 \mathrm{~mL})$ and piperidine $(1.3 \mathrm{~mL}, 12.8 \mathrm{mmol})$. Extraction and column chromatography $\left(\mathrm{SiO}_{2}\right.$, cyclohexane/EtOAc $\left.40: 1 \rightarrow 2: 1,3 \times 15 \mathrm{~cm}\right)$ yielded the product 15 (1.53 g, $5.06 \mathrm{mmol}, 80 \%)$ as a colorless solid. Mp 55-57 ${ }^{\circ} \mathrm{C} ;{ }^{1} \mathrm{H}-\mathrm{NMR}\left(400 \mathrm{MHz}, \mathrm{CDCl}_{3}\right), \delta$ (ppm): 8.47 (s, $1 \mathrm{H}, \mathrm{Ar}-H), 4.37$ (q, $\left.J_{H H} 7.1 \mathrm{~Hz}, 2 \mathrm{H}, \mathrm{CH}_{2} \mathrm{CH}_{3}\right), 3.77-3.71(\mathrm{~m}, 1 \mathrm{H}, \mathrm{CH}), 2.91-2.82$ $\left(\mathrm{m}, 2 \mathrm{H}, \mathrm{CH}_{2}\right), 2.76\left(\mathrm{~s}, 3 \mathrm{H}, \mathrm{CH}_{3}\right), 2.54-2.46\left(\mathrm{~m}, 2 \mathrm{H}, \mathrm{CH}_{2}\right), 2.45-2.37$ (m, 2H, CH$\left.)_{2}\right), 2.13-2.03$ (m, 2H, $\mathrm{CH}_{2}$ ), 2.03-1.94 (m, 1H, $\left.\mathrm{CH}_{2}\right), 1.79-1.64\left(\mathrm{~m}, 1 \mathrm{H}, \mathrm{CH}_{2}\right), 1.65-1.49$ (m, 6H, $\left.3 \times \mathrm{CH}_{2}\right), 1.49-$ $1.42\left(\mathrm{~m}, 1 \mathrm{H}, \mathrm{CH}_{2}\right), 1.39\left(\mathrm{q}, J_{H H} 7.1 \mathrm{~Hz}, 3 \mathrm{H}, \mathrm{CH}_{2} \mathrm{CH}_{3}\right) ;{ }^{13} \mathrm{C}\left\{{ }^{1} \mathrm{H}\right\}-\mathrm{NMR}\left(101 \mathrm{MHz}, \mathrm{CDCl}_{3}\right), \delta$ 
(ppm): 167.7 (s, $C=\mathrm{O}$ ), 161.2 (s, Ar-C), 157.2 (s, Ar- $C$ ), 138.7 (s, Ar- $C H$ ), 132.2 (s, Ar- $C$ ), 123.8 $(\mathrm{s}, \mathrm{Ar}-\mathrm{C}), 63.3(\mathrm{~s}, \mathrm{CH}), 61.3\left(\mathrm{~s}, \mathrm{CH}_{2} \mathrm{CH}_{3}\right), 50.1\left(\mathrm{~s}, 2 \times \mathrm{CH}_{2}\right), 33.2\left(\mathrm{~s}, \mathrm{CH}_{2}\right), 27.1\left(\mathrm{~s}, 2 \times C \mathrm{H}_{2}\right), 25.2$ (s, $\left.\mathrm{CH}_{2}\right), 24.7\left(\mathrm{~s}, \mathrm{CH}_{3}\right), 21.6\left(\mathrm{~s}, \mathrm{CH}_{2}\right), 21.0\left(\mathrm{~s}, \mathrm{CH}_{2}\right), 14.7\left(\mathrm{~s}, \mathrm{CH}_{2} \mathrm{CH}_{3}\right)$; EA $\left(\mathrm{C}_{18} \mathrm{H}_{26} \mathrm{~N}_{2} \mathrm{O}_{2}\right)$ : calc.: C 71.49, H 8.67, N 9.26; found: C 71.39, H 8.59, N 9.18; MS (EI, $\left.70 \mathrm{eV}, 150{ }^{\circ} \mathrm{C}\right) \mathrm{m} / \mathrm{z}(\%): 302$ (44) $[\mathrm{M}]^{+}, 273$ (35), 259 (38), 245 (11), 217 (100) [M - $\left.\mathrm{C}_{5} \mathrm{H}_{10} \mathrm{~N}\right]^{+}, 201$ (12), 190 (18), 172 (13), 144 (16), 86 (12); IR (neat), $v_{\max }\left(\mathrm{cm}^{-1}\right) 2940 \mathrm{~m}, 2920 \mathrm{~m}, 1863 \mathrm{w}, 2362 \mathrm{w}, 1715 \mathrm{~s}, 1590 \mathrm{w}, 1550 \mathrm{~m}$, $1371 \mathrm{w}, 1251 \mathrm{~s}, 1147 \mathrm{~s}, 1107 \mathrm{~m}, 1081 \mathrm{~m}, 1054 \mathrm{~m}, 961 \mathrm{~m}$; TLC: $\mathrm{R}_{f} 0.20\left(\mathrm{SiO}_{2}\right.$, cyclohexane/EtOAc $2: 1)$.

Ethyl 2-methyl-5-morpholino-5,6,7,8-tetrahydroquinoline-3-carboxylate (16). The reaction was set up as according to the general procedure using $11(2.00 \mathrm{~g}, 6.38 \mathrm{mmol})$ in acetonitrile (30 $\mathrm{mL})$ and morpholine $(1.1 \mathrm{~mL}, 12.8 \mathrm{mmol})$. Extraction and column chromatography $\left(\mathrm{SiO}_{2}\right.$, cyclohexane/EtOAc 20:1 $\rightarrow$ 7:1, $2 \times 15 \mathrm{~cm})$ yielded the product $16(1.50 \mathrm{~g}, 4.93 \mathrm{mmol}, 78 \%)$ as a colorless solid. Mp 83-85 ${ }^{\circ} \mathrm{C} ;{ }^{1} \mathrm{H}-\mathrm{NMR}\left(400 \mathrm{MHz}, \mathrm{CDCl}_{3}\right), \delta(\mathrm{ppm}): 8.44$ (s, $\left.1 \mathrm{H}, \mathrm{Ar}-H\right), 4.37$ (q, $\left.J_{H H} 7.1 \mathrm{~Hz}, 2 \mathrm{H}, \mathrm{CH}_{2} \mathrm{CH}_{3}\right), 3.80-3.66\left(\mathrm{~m}, 5 \mathrm{H}, \mathrm{CH}, 2 \times \mathrm{CH}_{2}\right), 2.93-2.84\left(\mathrm{~m}, 2 \mathrm{H}, \mathrm{CH}_{2}\right), 2.75$ (s, $\left.3 \mathrm{H}, \mathrm{CH}_{3}\right), 2.62-2.46\left(\mathrm{~m}, 4 \mathrm{H}, 2 \times \mathrm{CH}_{2}\right), 2.16-2.06\left(\mathrm{~m}, 1 \mathrm{H}, \mathrm{CH}_{2}\right), 2.04-1.95\left(\mathrm{~m}, 1 \mathrm{H}, \mathrm{CH}_{2}\right), 1.41(\mathrm{t}$, $\left.J_{H H} 7.1 \mathrm{~Hz}, 3 \mathrm{H}, \mathrm{CH}_{2} \mathrm{CH}_{3}\right) ;{ }^{13} \mathrm{C}\left\{{ }^{1} \mathrm{H}\right\}-\mathrm{NMR}\left(101 \mathrm{MHz}, \mathrm{CDCl}_{3}\right), \delta(\mathrm{ppm}): 167.5(\mathrm{~s}, C=\mathrm{O}), 161.5$ (s, $\operatorname{Ar}-C$ ), 157.6 (s, Ar-C),138.9 (s, Ar-CH), 131.0 (s, Ar-C), 123.8 (s, Ar-C), 68.0 (s, $\mathrm{CH}_{2} \mathrm{CH}_{3}$ ), $62.6(\mathrm{~s}, C \mathrm{H}), 61.4\left(\mathrm{~s}, 2 \times \mathrm{CH}_{2}\right), 49.3\left(\mathrm{~s}, 2 \times C \mathrm{H}_{2}\right), 33.1\left(\mathrm{~s}, C \mathrm{H}_{2}\right), 24.8\left(\mathrm{~s}, C \mathrm{H}_{2}\right), 21.3\left(\mathrm{~s}, C \mathrm{H}_{2}\right)$, $21.2\left(\mathrm{~s}, \mathrm{CH}_{3}\right), 14.7\left(\mathrm{~s}, \mathrm{CH}_{2} \mathrm{CH}_{3}\right)$; EA $\left(\mathrm{C}_{17} \mathrm{H}_{24} \mathrm{~N}_{2} \mathrm{O}_{3}\right)$ : calc.: $\mathrm{C}$ 67.08, H 7.95, N 9.20; found: $\mathrm{C}$ 67.24, H 7.89, N 9.22; MS (EI, $\left.70 \mathrm{eV}, 150{ }^{\circ} \mathrm{C}\right) \mathrm{m} / \mathrm{z}(\%): 304$ (24) [M] ${ }^{+}, 275$ (10), 259 (11), 217(100) [M-C $\left.{ }_{4} \mathrm{H}_{8} \mathrm{NO}\right]^{+}, 190$ (20), 172 (16), 144 (17), 86 (20); IR (neat), $v_{\max }\left(\mathrm{cm}^{-1}\right)$ 2955w, $2840 \mathrm{w}, 2361 \mathrm{w}, 1715 \mathrm{~s}, 1550 \mathrm{~m}, 1439 \mathrm{~m}, 1357 \mathrm{w}, 1325 \mathrm{w}, 1254 \mathrm{~s}, 1153 \mathrm{~m}, 1129 \mathrm{~m}, 1114 \mathrm{~s}, 1086 \mathrm{w}$, $1057 \mathrm{~m}, 1017 \mathrm{~m}$; TLC: $\mathrm{R}_{f} 0.19$ ( $\mathrm{SiO}_{2}$, cyclohexane/EtOAc 3:1).

Ethyl 5-((R)-2-(hydroxymethyl)pyrrolidin-1-yl)-2-methyl-5,6,7,8-tetrahydroquinoline-3carboxylate (17). The reaction was set up according to the general procedure using 11 (1.0 g, 3.19 mmol, 1.0 equiv.) in acetonitrile $(15 \mathrm{~mL})$ and L-prolinol ( $0.37 \mathrm{~mL}, 3.83 \mathrm{mmol}, 1.2$ equiv.). Extraction and column chromatography $\left(\mathrm{SiO}_{2}\right.$, cyclohexane / EtOAc 3:1 $\left.\rightarrow 1: 1,2 \times 25 \mathrm{~cm}\right)$ yielded a 1:1 mixture of diastereoisomers 17a and 17b $(673 \mathrm{mg}, 2.11 \mathrm{mmol}, 63 \%$ combined yield) as colorless oil. For characterization pure samples of $\mathbf{1 7 a}$ and $\mathbf{1 7 b}$ were prepared by chromatographic separation.

17a. ${ }^{1} \mathrm{H}-\mathrm{NMR}\left(400 \mathrm{MHz}, \mathrm{CDCl}_{3}\right), \delta$ (ppm): 8.30 (s, $\left.1 \mathrm{H}, \mathrm{Ar}-\mathrm{H}\right), 4.42-4.30\left(\mathrm{~m}, 2 \mathrm{H}, \mathrm{CH}_{2} \mathrm{CH}_{3}\right.$ ), 4.09-4.03 (m, $1 \mathrm{H}, \mathrm{CH}), 3.64\left(\mathrm{dd}, J_{H H} 10.8 \mathrm{~Hz}, J_{H H} 4.0 \mathrm{~Hz}, 1 \mathrm{H}, \mathrm{CH}_{2}-\mathrm{OH}\right), 3.46$ (dd, $J_{H H} 10.8 \mathrm{~Hz}$, $\left.J_{H H} 2.7 \mathrm{~Hz}, 1 \mathrm{H}, \mathrm{CH}_{2}-\mathrm{OH}\right), 3.19(\mathrm{~m}, 1 \mathrm{H}, \mathrm{CH}), 2.94-2.88\left(\mathrm{~m}, 2 \mathrm{H}, \mathrm{CH}_{2}\right), 2.77\left(\mathrm{~s}, 3 \mathrm{H}, \mathrm{CH}_{3}\right), 2.67-$ $2.58\left(\mathrm{~m}, 1 \mathrm{H}, \mathrm{CH}_{2}\right), 2.52-2.43\left(\mathrm{~m}, 1 \mathrm{H}, \mathrm{CH}_{2}\right), 2.16-2.08\left(\mathrm{~m}, 1 \mathrm{H}, \mathrm{CH}_{2}\right), 2.06-1.97\left(\mathrm{~m}, 1 \mathrm{H}, \mathrm{CH}_{2}\right)$, 1.97-1.81 (m, $\left.1 \mathrm{H}, \mathrm{CH}_{2}\right), 1.80-1.64\left(\mathrm{~m}, 4 \mathrm{H}, 2 \times \mathrm{CH}_{2}\right), 1.39\left(\mathrm{t}, J_{H H} 7.1 \mathrm{~Hz}, 3 \mathrm{H}, \mathrm{CH}_{2} \mathrm{CH}_{3}\right)$; ${ }^{13} \mathrm{C}\left\{{ }^{1} \mathrm{H}\right\}$-NMR $\left(101 \mathrm{MHz}, \mathrm{CDCl}_{3}\right), \delta(\mathrm{ppm}): 167.4(\mathrm{~s}, C=\mathrm{O}), 161.0(\mathrm{~s}, \mathrm{Ar}-C), 157.4(\mathrm{~s}, \mathrm{Ar}-C)$, 138.4 (s, Ar- $C H$ ), 132.0 (s, $\operatorname{Ar}-C$ ), 124.0 (s, $\operatorname{Ar}-C$ ), 62.6 (s, $\left.C_{2} \mathrm{CH}_{3}\right), 61.1$ (s, $\left.C \mathrm{H}_{2}\right), 60.0$ (s, $C H), 56.1(\mathrm{~s}, C \mathrm{H}), 46.6\left(\mathrm{~s}, C \mathrm{H}_{2}\right), 32.8\left(\mathrm{~s}, C \mathrm{H}_{2}\right), 28.9\left(\mathrm{~s}, C \mathrm{H}_{2}\right), 24.4\left(\mathrm{~s}, C \mathrm{H}_{2}\right), 24.3\left(\mathrm{~s}, C \mathrm{H}_{3}\right), 20.9$ $\left(\mathrm{s}, \mathrm{CH}_{2}\right), 20.8\left(\mathrm{~s}, \mathrm{CH}_{2}\right), 14.3\left(\mathrm{~s}, \mathrm{CH}_{2} \mathrm{CH}_{3}\right)$; EA $\left(\mathrm{C}_{18} \mathrm{H}_{26} \mathrm{~N}_{2} \mathrm{O}_{3}\right)$ : calc.: $\mathrm{C} \mathrm{67.90,} \mathrm{H} \mathrm{8.23,} \mathrm{N} \mathrm{8.80;}$ found: C 67.71, H 8.12, N 8.75; MS (FAB NBA) $m / z(\%): 319$ (96) [M] $]^{+}, 287$ (54), 218 (100) 
[M-C $\left.{ }_{5} \mathrm{H}_{10} \mathrm{NO}\right]^{+}, 190$ (15); IR (neat): $v_{\max }\left(\mathrm{cm}^{-1}\right) 3350 \mathrm{~b}, 2928 \mathrm{w}, 2860 \mathrm{w}, 1718 \mathrm{~s}, 1593 \mathrm{w}, 1555 \mathrm{w}$, $1443 \mathrm{~m}, 1365 \mathrm{w}, 1259 \mathrm{~s}, 1154 \mathrm{w}, 1059 \mathrm{~m}, 784 \mathrm{w} ;$ TLC $\mathrm{R}_{f} 0.42\left(\mathrm{SiO}_{2}\right.$, cyclohexane/EtOAc 1:1); $[\alpha]_{\mathrm{D}}^{20}:-112.5$ (c $\left.1.08, \mathrm{DCM}\right)$.

17b. ${ }^{1} \mathrm{H}-\mathrm{NMR}\left(400 \mathrm{MHz}, \mathrm{CDCl}_{3}\right), \delta(\mathrm{ppm}): 8.24(\mathrm{~s}, 1 \mathrm{H}, \mathrm{Ar}-H), 4.37$ (q, $J_{H H} 7.1 \mathrm{~Hz}, 2 \mathrm{H}$, $\left.\mathrm{CH}_{2} \mathrm{CH}_{3}\right), 4.10-4.06(\mathrm{~m}, 1 \mathrm{H}, \mathrm{CH}), 3.33-3.25\left(\mathrm{~m}, 3 \mathrm{H}, \mathrm{CH}_{2}-\mathrm{OH}, \mathrm{CH}\right), 2.98-2.93\left(\mathrm{~m}, 1 \mathrm{H}, \mathrm{CH}_{2}\right)$, 2.92-2.85 (m, 2H, CH $), 2.77\left(\mathrm{~s}, 3 \mathrm{H}, \mathrm{CH}_{3}\right), 2.74-2.64\left(\mathrm{~m}, 2 \mathrm{H}, \mathrm{CH}_{2}\right), 2.14-2.05\left(\mathrm{~m}, 1 \mathrm{H}, \mathrm{CH}_{2}\right)$, 2.04-1.89 (m, 2H, CH$), 1.87-1.69\left(\mathrm{~m}, 5 \mathrm{H}, 3 \times \mathrm{CH}_{2}\right), 1.40\left(\mathrm{t}, J_{H H} 7.1 \mathrm{~Hz}, 3 \mathrm{H}, \mathrm{CH}_{2} \mathrm{CH}_{3}\right)$; ${ }^{13} \mathrm{C}\left\{{ }^{1} \mathrm{H}\right\}$-NMR $\left(101 \mathrm{MHz}, \mathrm{CDCl}_{3}\right), \delta$ (ppm): 166.7 (s, $\left.C=\mathrm{O}\right), 161.1$ (s, Ar-C), 157.4 (s, Ar-C), 138.9 (s, Ar- $C H$ ), 131.1 (s, Ar- $C$ ), 122.7 (s, $\mathrm{Ar}-C), 61.7(\mathrm{~s}, C \mathrm{H}), 61.1\left(\mathrm{~s}, \mathrm{CH}_{2} \mathrm{CH}_{3}\right), 60.0(\mathrm{~s}, 2 \times$ $\left.C \mathrm{H}_{2}\right), 32.8\left(\mathrm{~s}, C \mathrm{H}_{2}\right), 29.2\left(\mathrm{~s}, 2 \times \mathrm{CH}_{2}\right), 27.0\left(\mathrm{~s}, C \mathrm{H}_{2}\right), 24.4\left(\mathrm{~s}, \mathrm{CH}_{2}\right), 24.4\left(\mathrm{~s}, C \mathrm{H}_{3}\right), 20.9\left(\mathrm{~s}, C \mathrm{H}_{2}\right)$, $14.3\left(\mathrm{~s}, \mathrm{CH}_{2} \mathrm{CH}_{3}\right)$; EA $\left(\mathrm{C}_{18} \mathrm{H}_{26} \mathrm{~N}_{2} \mathrm{O}_{3}\right)$ : calc.: C 67.90, $\mathrm{H} 8.23, \mathrm{~N} 8.80$; found: $\mathrm{C} 67.65, \mathrm{H} 8.15, \mathrm{~N}$ 8.76; MS (FAB NBA) m/z (\%): 319 (100) [M] ${ }^{+}, 287$ (53), 218 (86) [M-C $\left.{ }_{5} \mathrm{H}_{10} \mathrm{NO}\right]^{+}, 190$ (14); IR (neat): $v_{\max }\left(\mathrm{cm}^{-1}\right) 3355 \mathrm{~b}, 2936 \mathrm{w}, 2866 \mathrm{w}, 1717 \mathrm{~s}, 1594 \mathrm{w}, 1559 \mathrm{w}, 1442 \mathrm{~m}, 1363 \mathrm{w}, 1259 \mathrm{~s}, 1155 \mathrm{w}$, 1059m, 783w, 665m; TLC R $0.38\left(\mathrm{SiO}_{2}\right.$, cyclohexane/EtOAc 1:1); $[\alpha]_{\mathrm{D}}{ }^{20}:+26.8$ (c 1.06, DCM).

General procedure for the protonation with $\mathbf{H B F}_{4}$. To a solution of the amine (1.00 eq.) in $\mathrm{Et}_{2} \mathrm{O}(0.2 \mathrm{M})$ was added $\mathrm{HBF}_{4} \cdot \mathrm{Et}_{2} \mathrm{O}$ (0.95 eq.) dropwise. The mixture was stirred for $10 \mathrm{~min}$ at room temperature. The solvent was removed in vacuo and the residue was washed with dry $n$ pentane $(2 \times 2 \mathrm{~mL})$ to obtain the desired compound. All salts were shown to be unstable on both silica and alumina, and therefore precipitation was used for purification. The ${ }^{1} \mathrm{H},{ }^{13} \mathrm{C}$ and ${ }^{19} \mathrm{~F}$ spectra matched with the expected data, but the $\% \mathrm{C}$ values obtained by elemental analysis deviated from the calculated values by $1-2 \%$.

1-(3-(Ethoxycarbonyl)-2-methyl-5,6,7,8-tetrahydroquinolin-5-yl)pyrrolidin-1-ium 4-tetrafluoroborate (24). The reaction was set up according to the general procedure using 14 (150 $\mathrm{mg}$, $0.52 \mathrm{mmol}, 1.00$ equiv.) in $\mathrm{Et}_{2} \mathrm{O}(2.5 \mathrm{~mL})$ and $\mathrm{HBF}_{4} \cdot \mathrm{Et}_{2} \mathrm{O}(66.8 \mu \mathrm{L}, 0.50 \mathrm{mmol}, 0.95$ equiv. $)$. The title compound 24 (190 mg, $0.50 \mathrm{mmol}, 97 \%)$ was obtained as colorless solid. Mp 184-187 ${ }^{\circ} \mathrm{C}$, ${ }^{1} \mathrm{H}-\mathrm{NMR}$ (400 MHz, DMSO), $\delta$ (ppm): 9.39 (br s, 1H, NH), 8.42 (s, 1H, Ar-H), 4.74-4.67 (m, $1 \mathrm{H}, \mathrm{CH}), 4.41-4.26\left(\mathrm{~m}, 2 \mathrm{H}, \mathrm{CH}_{2} \mathrm{CH}_{3}\right), 3.58-3.48\left(\mathrm{~m}, 1 \mathrm{H}, \mathrm{CH}_{2}\right), 3.45-3.33\left(\mathrm{~m}, 1 \mathrm{H}, \mathrm{CH}_{2}\right), 3.28-$ $3.19\left(\mathrm{~m}, 1 \mathrm{H}, \mathrm{CH}_{2}\right), 3.15-2.98\left(\mathrm{~m}, 2 \mathrm{H}, \mathrm{CH}_{2}\right), 2.94-2.83\left(\mathrm{~m}, 1 \mathrm{H}, \mathrm{CH}_{2}\right), 2.73\left(\mathrm{~s}, 3 \mathrm{H}, \mathrm{CH}_{3}\right), 2.28-$

$2.17\left(\mathrm{~m}, 1 \mathrm{H}, \mathrm{CH}_{2}\right), 2.27-1.90\left(\mathrm{~m}, 4 \mathrm{H}, \mathrm{CH}_{2}\right), 1.96-1.69\left(\mathrm{~m}, 3 \mathrm{H}, \mathrm{CH} H_{2}\right), 1.33\left(\mathrm{t}, J_{H H} 7.1 \mathrm{~Hz}, 3 \mathrm{H}\right.$, $\left.\mathrm{CH}_{2} \mathrm{CH}_{3}\right) ;{ }^{13} \mathrm{C}\left\{{ }^{1} \mathrm{H}\right\}-\mathrm{NMR}$ (101 MHz, DMSO), $\delta$ (ppm): 166.0 (s, C=O), 161.4 (s, Ar-C), 159.2

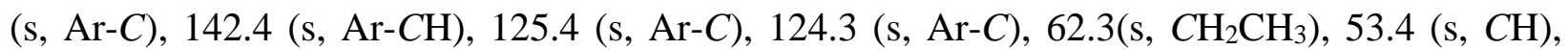
$51.5\left(\mathrm{~s}, 2 \times \mathrm{CH}_{2}\right), 31.2\left(\mathrm{~s}, C \mathrm{H}_{2}\right), 24.2\left(\mathrm{~s}, 2 \times C \mathrm{H}_{2}\right), 23.5\left(\mathrm{~s}, C \mathrm{H}_{2}\right), 23.4\left(\mathrm{~s}, C \mathrm{H}_{2}\right), 17.5\left(\mathrm{~s}, C \mathrm{H}_{3}\right)$, 15.0 (s, $\left.\mathrm{CH}_{2} \mathrm{CH}_{3}\right) ;{ }^{19} \mathrm{~F}\left\{{ }^{1} \mathrm{H}\right\}-\mathrm{NMR}$ (376 MHz, DMSO), $\delta$ (ppm): -148.0 (s); $\mathrm{EA}\left(\mathrm{C}_{17} \mathrm{H}_{25} \mathrm{BF}_{4} \mathrm{~N}_{2} \mathrm{O}_{2}\right)$ : calc.: C 54.28, H 6.70, N 7.45; found: C 56.38, H 6.85, N 7.86; MS (FAB NBA) $m / z$ (\%):377 (16) $[\mathrm{M}]^{+}, 289(100)\left[\mathrm{M}-\mathrm{BF}_{4}\right]^{+}, 218$ (51); IR (neat): $v_{\max }\left(\mathrm{cm}^{-1}\right) 3177 \mathrm{w}, 2966 \mathrm{w}, 2362 \mathrm{w}, 1718 \mathrm{~m}$, $1704 \mathrm{w}, 1423 \mathrm{w}, 1262 \mathrm{~s}, 1150 \mathrm{~s}, 1096 \mathrm{w}, 1060 \mathrm{w}, 910 \mathrm{w}$.

\section{1-(3-(Ethoxycarbonyl)-2-methyl-5,6,7,8-tetrahydroquinolin-5-yl)piperidin-1-ium 4-tetra-}

fluoroborate (26). The reaction was set up according to the general procedure using 15 (880 $\mathrm{mg}$, $2.91 \mathrm{mmol})$ in $\mathrm{Et}_{2} \mathrm{O}(14 \mathrm{~mL})$ and $\mathrm{HBF}_{4} \cdot \mathrm{Et}_{2} \mathrm{O}(0.37 \mathrm{~mL}, 0.50 \mathrm{mmol})$. Evaporation and washing with $n$-pentane $(2 \times 2 \mathrm{~mL})$ yielded the title compound 26 (1.10 g, $2.82 \mathrm{mmol}, 97 \%)$. Mp 196-197 
${ }^{\circ} \mathrm{C} ;{ }^{1} \mathrm{H}-\mathrm{NMR}$ (400 MHz, DMSO), $\delta$ (ppm): 8.88 (br s, $\left.1 \mathrm{H}, \mathrm{NH}\right), 8.44$ (s, $1 \mathrm{H}, \mathrm{Ar}-H$ ), 4.83-4.76 (m, 1H, CH), 4.41-4.27 (m, 2H, $\left.\mathrm{CH}_{2} \mathrm{CH}_{3}\right), 3.40-3.32\left(\mathrm{~m}, 1 \mathrm{H}, \mathrm{CH}_{2}\right), 3.19-3.00\left(\mathrm{~m}, 2 \mathrm{H}, \mathrm{CH}_{2}\right)$, 3.00-2.88 (m, 2H, CH $), 2.96-2.75\left(\mathrm{~m}, 1 \mathrm{H}, \mathrm{CH}_{2}\right), 2.69\left(\mathrm{~s}, 3 \mathrm{H} \mathrm{CH} \mathrm{CH}_{3}, 2.23-1.96\left(\mathrm{~m}, 3 \mathrm{H}, \mathrm{CH}_{2}\right)\right.$, 1.89-1.49 (m, 6H, CH$), 1.33\left(\mathrm{t}, J_{H H} 7.1 \mathrm{~Hz}, 3 \mathrm{H}, \mathrm{CH}_{2} \mathrm{CH}_{3}\right) ;{ }^{13} \mathrm{C}\left\{{ }^{1} \mathrm{H}\right\}-\mathrm{NMR}$ (101 MHz, DMSO), $\delta$ (ppm): 167.7 (s, $C=\mathrm{O}$ ), 164.6 (s, Ar-C), 157.2 (s, Ar- $C$ ), 138.7 (s, Ar- $C H$ ), 124.8 (s, Ar-C), 124.3 (s, $\operatorname{Ar}-C), 61.7\left(\mathrm{~s}, \mathrm{CH}_{2} \mathrm{CH}_{3}\right), 61.2(\mathrm{~s}, C \mathrm{H}), 50.4\left(\mathrm{~s}, 2 \times C \mathrm{H}_{2}\right), 47.6\left(\mathrm{~s}, C \mathrm{H}_{2}\right), 22.9\left(\mathrm{~s}, 2 \times C \mathrm{H}_{2}\right)$, $22.7\left(\mathrm{~s}, C \mathrm{H}_{2}\right), 21.1\left(\mathrm{~s}, C \mathrm{H}_{2}\right), \quad 18.2\left(\mathrm{~s}, C \mathrm{H}_{2}\right), 15.2\left(\mathrm{~s}, C \mathrm{H}_{3}\right), 14.5\left(\mathrm{~s}, \mathrm{CH}_{2} C_{3}\right),{ }^{19} \mathrm{~F}\left\{{ }^{1} \mathrm{H}\right\}-\mathrm{NMR}$ (376 MHz, DMSO), $\delta$ (ppm): -148.0 (s); EA $\left(\mathrm{C}_{18} \mathrm{H}_{27} \mathrm{BF}_{4} \mathrm{~N}_{2} \mathrm{O}_{2}\right)$ : calc.: C 55.40, H 6.97, N 7.18; found: C 56.56, H 6.93, N 7.21; MS (FAB NBA) $m / z(\%): 303$ (100) [M -BF $]^{+}, 218$ (55) [M $\left.\mathrm{BF}_{4},-\mathrm{C}_{5} \mathrm{H}_{10} \mathrm{~N}\right]^{+}$; IR (neat): $v_{\max }\left(\mathrm{cm}^{-1}\right) 3154 \mathrm{w}, 2948 \mathrm{w}, 1721 \mathrm{~m}, 1558 \mathrm{w}, 1447 \mathrm{w}, 1263 \mathrm{~m}, 1213 \mathrm{w}$, 1144w, 1059s, 999s, 784w, 684w.

1-(3-(Ethoxycarbonyl)-2-methyl-5,6,7,8-tetrahydroquinolin-5-yl)morpholin-1-ium tetrafluoroborate (27). The reaction was set up as in the general procedure except using 16 (80.0 mg, $0.26 \mathrm{mmol}$, 1.00 equiv.) in $\mathrm{Et}_{2} \mathrm{O}(1.5 \mathrm{~mL})$ was added $\mathrm{HBF}_{4} \cdot \mathrm{Et}_{2} \mathrm{O}(34 \mu \mathrm{L}, 0.25 \mathrm{mmol}$, 0.95 equiv.).Evaporation and washing with $n$-pentane $(2 \times 2 \mathrm{~mL})$ yielded the title compound 27 (97.0 mg, $0.25 \mathrm{mmol}, 97 \%$ ) as colorless solid. Mp $252{ }^{\circ} \mathrm{C} .{ }^{1} \mathrm{H}-\mathrm{NMR}$ (400 MHz, DMSO): $\delta / \mathrm{ppm}$ 9.37 (br s, $1 \mathrm{H}, \mathrm{NH}), 8.36$ (s, $1 \mathrm{H}, \mathrm{Ar}-\mathrm{H}), 4.75$ (s, $1 \mathrm{H}, \mathrm{CH}), 4.41-4.25\left(\mathrm{~m}, 2 \mathrm{H}, \mathrm{CH}_{2} \mathrm{CH}_{3}\right), 4.02-3.89$ (m, 2H, $\mathrm{CH}_{2}$ ), 3.72-3.57 (m, 2H, CH$), 3.40-3.19$ (m, 2H, $\left.\mathrm{CH}_{2}\right), 3.19-3.06$ (m, 1H, CH $)$, 3.06$2.95\left(\mathrm{~m}, 1 \mathrm{H}, \mathrm{CH}_{2}\right), 2.92-2.77\left(\mathrm{~m}, 1 \mathrm{H}, \mathrm{CH}_{2}\right), 2.71(\mathrm{~s}, 3 \mathrm{H} \mathrm{CH} 3), 2.38-2.22\left(\mathrm{~m}, 1 \mathrm{H}, \mathrm{CH}_{2}\right), 2.07-$ $1.93\left(\mathrm{~m}, 1 \mathrm{H}, \mathrm{CH}_{2}\right), 1.88-1.73(\mathrm{~m}, 1 \mathrm{H}, \mathrm{CH}), 1.33\left(\mathrm{t}, J_{H H} 7.1 \mathrm{~Hz}, 3 \mathrm{H}, \mathrm{CH}_{2} \mathrm{CH}_{3}\right) .{ }^{13} \mathrm{C}\left\{{ }^{1} \mathrm{H}\right\}-\mathrm{NMR}$ (101 MHz, DMSO): $\delta /$ ppm 165.0 (s, $C=\mathrm{O}$ ), 160.4 (s, Ar-C), 159.7 (s, Ar-C), 145.5 (s, Ar- $C H$ ), $131.0(\mathrm{~s}, \mathrm{Ar}-\mathrm{C}), 125.60(\mathrm{~s}, \mathrm{Ar}-\mathrm{C}), 62.7\left(\mathrm{~s}, \mathrm{CH}_{2} \mathrm{CH}_{3}\right), 62.2(\mathrm{~s}, C \mathrm{H}), 52.4\left(\mathrm{~s}, 2 \times \mathrm{CH}_{2}\right), 50.6(\mathrm{~s}$, $\left.2 \times \mathrm{CH}_{2}\right), 29.7\left(\mathrm{~s}, C \mathrm{H}_{2}\right), 22.5\left(\mathrm{~s}, C \mathrm{H}_{2}\right), 21.7\left(\mathrm{~s}, C \mathrm{H}_{2}\right), 17.8\left(\mathrm{~s}, C \mathrm{H}_{3}\right), 14.7\left(\mathrm{~s}, \mathrm{CH}_{2} C_{3}\right) .{ }^{19} \mathrm{~F}\left\{{ }^{1} \mathrm{H}\right\}-$ NMR (376 MHz, DMSO): $\delta / p p m-148.0$ (s). EA $\left(\mathrm{C}_{17} \mathrm{H}_{24} \mathrm{BF}_{4} \mathrm{~N}_{2} \mathrm{O}_{3}\right):$ C 52.20, H 6.18, N 7.16; found: C 52.45, H 6.11, N 7.28. MS (FAB NBA) $m / z(\%): 305$ (100) $\left[\mathrm{MH}-\mathrm{BF}_{4}\right]^{+}, 218(54)$ $\left[\mathrm{MH}-\mathrm{C}_{4} \mathrm{H}_{8} \mathrm{NO}\right]^{+}, 190$ (11), 149 (15). IR (neat): $\tilde{v} / \mathrm{cm}^{-1} 3110 \mathrm{w}, 1726 \mathrm{~s}, 1641 \mathrm{~m}, 1407 \mathrm{w}, 1291 \mathrm{~m}$, 1101s, 1080s, 969s, 920m, 764m, 677w.

General procedure for the methylation and deprotonation of the protonated amino pyridines 3. The protonated amine (1.0 eq.) was dissolved in dioxane $(0.02 \mathrm{M})$ and treated with methyl trifluoromethanesulfonate (1.0 eq.). The mixture was stirred for 4 hours at room temperature. The solvent was removed in vacuo and the resulting brown residue was washed with dry $n$-pentane $(2 \times 2 \mathrm{~mL})$ to obtain the corresponding biscationic salt. The crude mixture was dissolved in dioxane $(0.2 \mathrm{M})$ and sodium hydride (1.5 equiv.) was added. The mixture was stirred for $30 \mathrm{~min}$ at $\mathrm{rt}$. The obtained yellow solution was concentrated in vacuo and washed with dry $n$-pentane $(2 \times 30 \mathrm{~mL})$. The resulting solid material was dissolved in DCM $(2 \mathrm{~mL})$ and filtered through a disposable HPLC filter $\left(\right.$ Chromafill $^{\circledR} 0-20 / 15 \mathrm{MS}$, pore size $20 \mu \mathrm{m}$ ) to remove insoluble salts. Evaporation of the solvent yielded the title compound.

3-(Ethoxycarbonyl)-1,2-dimethyl-5-(pyrrolidin-1-yl)-5,6,7,8-tetrahydroquinolin-1-ium trifluoromethanesulfonate (30). The reaction was set up according to the general procedure using 24 (250 mg, $0.66 \mathrm{mmol})$, methyl trifluoromethanesulfonate $(112 \mathrm{mg}, 73 \mu \mathrm{L}, 0.66 \mathrm{mmol})$ and 
sodium hydride $(25 \mathrm{mg}, 0.99 \mathrm{mmol})$. Compound 30 was obtained as a red oil $(190 \mathrm{mg}$, $0.42 \mathrm{mmol}, 64 \%) .{ }^{1} \mathrm{H}-\mathrm{NMR}\left(400 \mathrm{MHz}, \mathrm{CDCl}_{3}\right), \delta(\mathrm{ppm}): 8.67$ (s, $\left.1 \mathrm{H}, \mathrm{Ar}-H\right), 4.47-4.37$ (m, 2H, $\left.\mathrm{CH}_{2} \mathrm{CH}_{3}\right), 4.06\left(\mathrm{~s}, 3 \mathrm{H}, \mathrm{N}-\mathrm{CH}_{3}\right), 3.91(\mathrm{~s}, 1 \mathrm{H}, \mathrm{CH}), 3.25-3.06\left(\mathrm{~m}, 2 \mathrm{H}, \mathrm{CH}_{2}\right), 2.91\left(\mathrm{~s}, 3 \mathrm{H}, \mathrm{CH}_{3}\right), 2.65-$ $2.56\left(\mathrm{~m}, 2 \mathrm{H}, \mathrm{CH}_{2}\right), 2.47-2.40\left(\mathrm{~m}, 2 \mathrm{H}, \mathrm{CH}_{2}\right), 2.24-2.12\left(\mathrm{~m}, 1 \mathrm{H}, \mathrm{CH}_{2}\right), 1.91-1.76\left(\mathrm{~m}, 3 \mathrm{H}, \mathrm{CH}_{2}\right)$, 1.75-1.64 (m, 4H, CH$\left.H_{2}\right), 1.35$ (t, $\left.J_{H H} 7.1 \mathrm{~Hz}, 3 \mathrm{H}, \mathrm{CH}_{2} \mathrm{CH}_{3}\right) ;{ }^{13} \mathrm{C}\left\{{ }^{1} \mathrm{H}\right\}-\mathrm{NMR}\left(101 \mathrm{MHz}, \mathrm{CDCl}_{3}\right), \delta$ (ppm): 164.1 (s, $C=\mathrm{O}$ ), 158.3 (s, Ar-C), 154.8 (s, Ar-C), 142.6 (s, Ar-CH), 137.5 (s, Ar-C), $123.0(\mathrm{~s}, \mathrm{Ar}-\mathrm{C}), 62.6\left(\mathrm{~s}, \mathrm{CH}_{2} \mathrm{CH}_{3}\right), 57.8(\mathrm{~s}, \mathrm{CH}), 49.0\left(\mathrm{~s}, 2 \times \mathrm{CH}_{2}\right), 40.6\left(\mathrm{~s}, \mathrm{~N}-\mathrm{CH}_{3}\right), 28.6(\mathrm{~s}$, $\left.\mathrm{CH}_{2}\right), 23.2$ (s, $\left.2 \times \mathrm{CH}_{2}\right), 20.6$ (s, $\left.\mathrm{CH}_{2}\right), 18.1\left(\mathrm{~s}, \mathrm{CH}_{3}\right), 17.6\left(\mathrm{~s}, \mathrm{CH}_{2}\right), 13.7\left(\mathrm{~s}, \mathrm{CH}_{2} \mathrm{CH}_{3}\right) ;{ }^{19} \mathrm{~F}\left\{{ }^{1} \mathrm{H}\right\}-$ NMR (376 MHz, $\left.\mathrm{CDCl}_{3}\right), \delta(\mathrm{ppm}):-77.4$ (s); EA $\left(\mathrm{C}_{19} \mathrm{H}_{27} \mathrm{~F}_{3} \mathrm{~N}_{2} \mathrm{O}_{5} \mathrm{~S}\right)$ : calc.: C 50.43, H 6.01, N

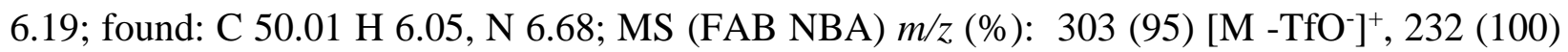
[M - $\left.\mathrm{TfO}^{-},-\mathrm{C}_{4} \mathrm{H}_{9} \mathrm{~N}\right]^{+}, 218$ (11), 204 (18); IR (neat): $v_{\max }\left(\mathrm{cm}^{-1}\right) 2980 \mathrm{w}, 2955 \mathrm{w}, 1719 \mathrm{~m}, 1455 \mathrm{w}$, $1320 \mathrm{~s}, 1262 \mathrm{~m}, 1196 \mathrm{~m}, 1140 \mathrm{~s}, 1100 \mathrm{~m}, 1057 \mathrm{~s}, 1015 \mathrm{~s}, 971 \mathrm{~m}, 842 \mathrm{w}, 738 \mathrm{~m}, 728 \mathrm{~m}$.

3-(Ethoxycarbonyl)-1,2-dimethyl-5-(piperidin-1-yl)-5,6,7,8-tetrahydroquinolin-1-ium trifluoromethanesulfonate (31). The reaction was set up according to the general procedure using $26(200 \mathrm{mg}, 0.51 \mathrm{mmol})$, methyl trifluoromethanesulfonate $(86.3 \mathrm{mg}, 60 \mu \mathrm{L}, 0.51 \mathrm{mmol})$ and sodium hydride $(20 \mathrm{mg}, 0.77 \mathrm{mmol})$. Compound 31 was obtained as a yellow oil (135 mg, $0.29 \mathrm{mmol}, 57 \%$ ). ${ }^{1} \mathrm{H}-\mathrm{NMR}\left(400 \mathrm{MHz}, \mathrm{CDCl}_{3}\right), \delta(\mathrm{ppm}): 8.93$ (s, $\left.1 \mathrm{H}, \mathrm{Ar}-H\right), 4.42$ (q, $J_{H H} 7.1 \mathrm{~Hz}$ , $\left.2 \mathrm{H}, \mathrm{CH}_{2} \mathrm{CH}_{3}\right), 4.05\left(\mathrm{~s}, 3 \mathrm{H}, \mathrm{N}-\mathrm{CH}_{3}\right), 4.00-3.91(\mathrm{~m}, 1 \mathrm{H}, \mathrm{CH}), 3.18-3.04\left(\mathrm{~m}, 1 \mathrm{H}, \mathrm{CH}_{2}\right), 2.94(\mathrm{~s}$, $\left.3 \mathrm{H}, \mathrm{CH}_{3}\right), 2.54-2.35\left(\mathrm{~m}, 6 \mathrm{H}, \mathrm{CH}_{2}\right), 2.26-2.10\left(\mathrm{~m}, 1 \mathrm{H}, \mathrm{CH}_{2}\right), 2.06-1.93\left(\mathrm{~m}, 1 \mathrm{H}, \mathrm{CH}_{2}\right), 1.89-1.65$ $\left(\mathrm{m}, 2 \mathrm{H}, \mathrm{CH}_{2}\right), 1.63-1.41\left(\mathrm{~m}, 6 \mathrm{H}, \mathrm{CH}_{2}\right), 1.36\left(\mathrm{t}, J_{H H} 7.1 \mathrm{~Hz}, 3 \mathrm{H}, \mathrm{CH}_{2} \mathrm{CH}_{3}\right) ;{ }^{13} \mathrm{C}\left\{{ }^{1} \mathrm{H}\right\}-\mathrm{NMR}(101$ $\mathrm{MHz}, \mathrm{CDCl}_{3}$ ), $\delta(\mathrm{ppm}): 164.1$ (s, $C=\mathrm{O}$ ), 158.3 (s, $\mathrm{Ar}-\mathrm{C}$ ), 154.7 (s, $\mathrm{Ar}-\mathrm{C}$ ), 142.6 (s, $\mathrm{Ar}-\mathrm{CH}$ ), 137.4 (s, $\mathrm{Ar}-C$ ), 128.2 (s, $\operatorname{Ar}-C), 62.5\left(\mathrm{~s}, \mathrm{CH}_{2} \mathrm{CH}_{3}\right), 61.4(\mathrm{~s}, \mathrm{CH}), 50.0\left(\mathrm{~s}, 2 \times C \mathrm{H}_{2}\right), 40.5(\mathrm{~s}, \mathrm{~N}-$ $\left.\mathrm{CH}_{3}\right), 28.8\left(\mathrm{~s}, \mathrm{CH}_{2}\right), 26.2\left(\mathrm{~s}, 2 \times \mathrm{CH}_{2}\right), 24.0\left(\mathrm{~s}, \mathrm{CH}_{2}\right), 19.5\left(\mathrm{~s}, \mathrm{CH}_{2}\right), 18.7\left(\mathrm{~s}, \mathrm{CH}_{3}\right), 18.1\left(\mathrm{~s}, \mathrm{CH}_{2}\right)$, $13.8\left(\mathrm{~s}, \mathrm{CH}_{2} \mathrm{CH}_{3}\right) ;{ }^{19} \mathrm{~F}\left\{{ }^{1} \mathrm{H}\right\}$-NMR $\left(376 \mathrm{MHz}, \mathrm{CDCl}_{3}\right), \delta(\mathrm{ppm})$ : -77.5 (s); $\mathrm{EA}\left(\mathrm{C}_{20} \mathrm{H}_{29} \mathrm{~F}_{3} \mathrm{~N}_{2} \mathrm{O}_{5} \mathrm{~S}\right)$ : calc.: C 51.49, H 6.27, N 6.00; found: C 51.08, H 6.33, N 6.23; MS (FAB NBA) $\mathrm{m} / z$ (\%): 317

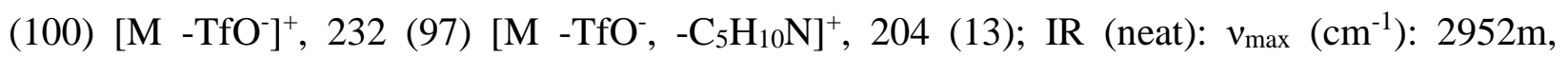
$2920 \mathrm{~m}, 1863 \mathrm{w}, 2362 \mathrm{w}, 1715 \mathrm{~s}, 1590 \mathrm{w}, 1550 \mathrm{~m}, 1371 \mathrm{w}, 1251 \mathrm{~s}, 1147 \mathrm{~s}, 1123 \mathrm{~m}, 1071 \mathrm{~m}, 886 \mathrm{~m}$.

\section{Acknowledgements}

Financial support from the Swiss National Science Foundation is gratefully acknowledged. P. M. thanks the European Union for a Marie Curie Intra European Fellowship (IEF). We thank Dr. Heinz Nadig (Department of Chemistry, University of Basel) for measuring the mass spectra and Mr. Werner Kirsch (Department of Chemistry University of Basel) for elemental analyses. 


\section{References}

1. Burgess, V. A.; Davies, S. G.; Skerlj, R. T. Tetrahedron Asymmetry 1991, 5, 299.

http://dx.doi.org/10.1016/S0957-4166(00)82109-6

2. Fu, Q.; Yan, C.-G. Tetrahedron 2013, 69, 5841.

http://dx.doi.org/10.1016/j.tet.2013.05.034

3. Pernak, J.; Rogoza, J. Arkivoc 2000, (vi), 889. http://dx.doi.org/10.3998/ark.5550190.0001.606

4. Kiamehr, M.; Moghaddam, F. M.; Gormay, P. V.; Semeniuchenko, V.; Villinger, A.; Langer, P.; Iaroshenko, V. O. Tetrahedron 2012, 68, 9685. http://dx.doi.org/10.1016/j.tet.2012.09.059

5. Litvić, M.; Filipan, M.; Pogorelić, I.; Cepanec, I. Green Chem. 2005, 7, 771. http://dx.doi.org/10.1039/b510276f

6. Pyrko, A. Chem. Heterocycl. Compds. 1999, 35, 688. http://dx.doi.org/10.1007/BF02251627

7. Uenishi, J.-i.; Hamada, M.; Aburatani, S.; Matsui, K.; Yonemitsu, O.; Tsukube, H. J. Org. Chem. 2004, 69, 6781.

http://dx.doi.org/10.1021/jo0491758

PMid:15387603

8. Clayden, N. P.; Greeves, N.; Warren, S.; Wothers, P. D. Organic Chemistry; Oxford University Press: Oxford, 2001.

http://dx.doi.org/10.1007/s00897010513a 